\title{
Numerical Simulation of Baroclinic Jovian Vortices *
}

\author{
Richard K. ACHTERBERG** AND ANDREW P. INGERSOLL \\ Division of Geological and Planetary Sciences, California Institute of Technology, Pasadena, California
}

(Manuscript received 3 February 1992, in final form 16 August 1993)

\begin{abstract}
We examine the evolution of baroclinic vortices in a time-dependent, nonlinear numerical model of a Jovian atmosphere. The model uses a normal-mode expansion in the vertical, using the barotropic and first two baroclinic modes. Results for the stability of baroclinic vortices on an $f$ plane in the absence of a mean zonal flow are similar to results of Earth vortex models, although the presence of a fiuid interior on the Jovian planets shifts the stability boundaries to smaller length scales. The presence of a barotropic mean zonal flow in the interior stabilizes vortices against instability and significantly modifies the finite amplitude form of baroclinic instabilities. The effect of a zonal flow on a form of barotropic instability produces periodic oscillations in the latitude and longitude of the vortex as observed at the level of the cloud tops. This instability may explain some, but not all, observations of longitudinal oscillations of vortices on the outer planets. Oscillations in aspect ratio and orientation of stable vortices in a zonal shear flow are observed in this baroclinic model, as in simpler twodimensional models. Such oscillations are also observed in the atmospheres of Jupiter and Neptune. The meridional propagation and decay of vortices on a $\beta$ plane is inhibited by the presence of a mean zonal flow. The direction of propagation of a vortex relative to the mean zonal flow depends upon the sign of the meridional potential vorticity gradient; combined with observations of vortex drift rates, this may provide a constraint on model assumption for the flow in the deep interior of the Jovian planets.
\end{abstract}

\section{Introduction}

One of the characteristic features of Jupiter's atmosphere is the large number of long-lived spots, ranging in size from the Great Red Spot (GRS), about 25000 $\mathrm{km}$ in length, down to the limit of resolution of the Voyager spacecraft (Smith et al. 1979a,b). Spots are also observed on Saturn (Ingersoll et al. 1984) and Neptune (Smith et al. 1989) but are not as common on these planets as on Jupiter. Ground-based and spacecraft observations have determined many properties that should be explained by theoretical models of the spots.

(i) Most spots are long-lived; the GRS has existed for over 300 years, and the White Ovals for over 50 years (Peek 1958), while the smaller spots observed by Voyager lasted for the duration of the observations ( $\sim 60$ days) unless they interacted with each other or with other features (Mac Low and Ingersoll 1986).

\footnotetext{
* Contribution No. 5114 from the Division of Geological and Planetary Sciences, California Institute of Technology, Pasadena, California.

* * Current affiliation: NASA Goddard Space Flight Center, Laboratory for Extraterrestrial Physics, Greenbelt, Maryland.
}

Corresponding author address: Dr. R. K. Achterberg, NASA Goddard Space Flight Center, Mail Code 693.2, Greenbelt, MD 20771. (ii) Spots exist in a mean zonal flow, with the vorticity of the zonal flow of the same sign as the vorticity of the spot. Where measured, the vorticity of the spots is greater than the vorticity of the mean zonal flow (Mitchell et al. 1981; Hatzes et al. 1981; Polvani et al. 1990).

(iii) Ninety percent of spots are anticyclonic (Mac Low and Ingersoll 1986).

(iv) Interactions between spots usually lead to merging of the spots (Mac Low and Ingersoll 1986).

(v) The smallest spots are nearly circular, while larger spots are more elliptical (Mac Low and Ingersoll 1986).

(vi) Some spots display periodic variations in their aspect ratio and in the orientation of their major axis. The total area of the spot is conserved during the oscillations. These oscillations have been observed in detail for a brown barge on Jupiter (Hatzes et al. 1981) and for the Great Dark Spot on Neptune (Smith et al. 1989; Polvani et al. 1990).

(vii) A few spots have been observed to oscillate in longitude about their mean motion. The best known example is Jupiter's Great Red Spot, which oscillates in longitude about its mean motion with a period of just under 90 days and an amplitude of about 1 deg (e.g., Reese 1972). This oscillation persists even when the long-term mean motion of the GRS changes. The spots on Neptune oscillate in both latitude and longitude, with peak-to-peak amplitudes up to $90 \mathrm{deg}$ in longitude (Hammel et al. 1989; Sromovsky 1991). 
Many models have been proposed to explain these spots but none of them explains all the available observations. Early models of the GRS postulated that it is the upper end of a Taylor column forced by a topographic feature on the surface (Hide 1961; Ingersoll 1969). Because current models of Jupiter indicate that there is no solid surface (e.g., Stevenson 1982), the Taylor column models are not viable.

Maxworthy and Redekopp (1976) model the GRS as a weakly nonlinear soliton in a zonal shear flow, using the quasigeostrophic approximation with rigid upper and lower boundaries. The vortex is maintained against dissipation by absorbing energy directly from the zonal flow, which is barotropically unstable. The interaction of two solitons results in their passing through each other unchanged except for a shift in position. This is contradicted by observations that vortex interactions usually lead to merging of the vortices (Mac Low and Ingersoll 1986).

The modon model of Ingersoll and Cuong (1981) uses a quasigeostrophic $11 / 2$-layer model, with a thin upper weather layer of constant density representing Jupiter's lower troposphere over an infinitely deep layer of slightly higher density representing Jupiter's fluid interior. The mean zonal flow is assumed the same in both layers, and the vortex is confined to the upper layer. If the length scales of both the flow and the vortex are larger than the radius of deformation, then the zonal flow is stable and there are exact, strongly nonlinear, steady and stable solutions in which the potential vorticity is a multivalued function of the streamfunction. In this model, as in the soliton model, nonlinear effects act to balance Rossby wave dispersion. Ingersoll and Cuong show that the interaction of two modon solutions results in their merging, and propose that large vortices on Jupiter are maintained against dissipation by the absorption of smaller vortices.

Williams and Yamagata, (1984) argue that the GRS and other large vortices are too large to be modeled by quasigeostrophic dynamics but instead are the fundamental solutions of an "intermediate geostrophic" regime. They show that anticyclonic vortices are more stable than cyclonic vortices in the intermediate geostrophic approximation and that the interaction between two intermediate geostrophic vortices results in the vortices merging. The difference between cyclonic and anticyclonic vortices, as well as the merging of vortices, has also been observed in laboratory experiments of vortices in the same dynamical regime (Nezlin et al. 1990). Williams and Yamagata propose that the GRS was formed by, and is maintained against dissipation by, a weak barotropic instability of the mean zonal flow. Formation of a GRS-like vortex by barotropic instability has also been demonstrated in numerical simulations by Williams and Wilson (1988) and Dowling and Ingersoll (1989), the latter using the observed zonal flow with bottom topography de- rived from a vorticity analysis of the GRS and White Oval BC.

Read and Hide $(1983,1984)$ propose that Jovian vortices result from baroclinic instability of the mean flow, based upon an analogy with laboratory experiments on sloping convection in an internally heated fluid in a rotating annulus. Furthermore, Read (1986) shows that vortices seen in numerical simulations of the annulus experiments are very nearly free-mode solutions of the inviscid equations of motion with the deviations from the free mode caused by the thermal forcing.

Marcus $(1988,1990)$ uses a 11/2-layer quasigeostrophic model with a mean zonal flow having constant background potential vorticity when averaged over large scales. He finds, using a numerical model, that patches of anomalous potential vorticity of the same sign as the vorticity of the background flow eventually merge to form a single large vortex embedded in a turbulent zonal flow. Similar behavior has been observed in laboratory experiments (Sommeria et al. 1988; Meyers et al. 1989).

Most of these models use the 11/2-layer model, with motions in the interior assumed to be a function only of latitude. Such a model is dynamically equivalent to a reduced gravity single-layer model with bottom topography (Gill 1982) and does not allow baroclinic processes, most notably baroclinic instability. Those models of Jovian atmospheric dynamics that are baroclinic (e.g., Maxworthy and Redekopp 1976; Read and Hide 1983, 1984) use a solid lower boundary instead of a fluid planetary interior, as do baroclinic models that have been used to study vortices in the Earth's atmosphere and oceans.

In this paper, we will be concerned primarily with the nonlinear stability and long-term survival of vortices in a quasigeostrophic, baroclinic model with two degrees of freedom in the vertical and an infinitely deep, neutrally stratified lower layer, representing the fluid interior of a Jovian planet. Of particular interest are the effects of using an infinitely deep, neutrally stratified lower layer instead of the solid lower boundary of previous baroclinic models, as well as the stability of equivalent barotropic vortices (those with the same vertical structure as in the single layer models) in a model that allows for a more complicated vertical structure. We also wish to investigate the conditions under which long-lived vortices can exist, and the use of vortices as a probe of atmospheric structure. If we find that a distinctive behavior occurs only for vortices within a certain size range relative to the deformation radius, then an observation of a vortex that exhibits that behavior can be used to constrain the radius of deformation. Similarly, vortices might also provide limits on the mean zonal velocity below the observed clouds.

We find that stable or apparently stable vortices exist over the range of sizes considered in our model. The replacement of the solid lower boundary with a fluid 
layer shifts the boundaries between stable and unstable regions to smaller length scales but does not change the qualitative behavior of the instabilities. We also find that equivalent barotropic vortices can be unstable in a baroclinic model if the diameter of the vortex is smaller than the deformation radius. Since this instability manifests itself as a growing oscillation, it may explain some observations of vortices that oscillate in longitude and thus provide limits on Jupiter's radius of deformation. We also find that the decay of vortices by the radiation of Rossby waves can be inhibited by the presence of the mean zonal flow. In addition, the direction that vortices propagate relative to the zonal flow depends upon the sign of the meridional potential vorticity gradient. Observations of vortex drift rates may thus be useful for testing assumptions about the zonal flow below the observed cloud layer.

This paper is organized as follows. In section 2 we briefly describe our numerical model, which uses a normal-mode expansion in the vertical. A more detailed derivation of the normal-mode expansion for Jovian atmospheres is given by Achterberg and Ingersoll (1989). Section 3 discusses the stability of baroclinic vortices on an $f$ plane (ignoring the meridional variation of the Coriolis parameter) in the absence of a mean zonal flow. The effects of adding a mean zonal flow upon the stability of vortices are examined in section 4. Section 5 looks at the effects of a meridional potential vorticity gradient (the $\beta$ effect). Comparison of the model with observations of the outer planets is done in section 6 .

\section{Model description}

Our model is based on the quasigeostrophic equation for the conservation of potential vorticity on the $\beta$ plane. This is given in log-pressure coordinates as (e.g., Pedlosky 1987)

$$
\frac{D}{D t}\left(\nabla^{2} \psi+\beta y+\mathscr{L} \psi\right)=0
$$

where

$$
\begin{aligned}
\mathscr{L} a & =e^{z} \frac{\partial}{\partial z}\left(\frac{e^{-z} f_{0}^{2}}{N^{2} H^{2}} \frac{\partial a}{\partial z}\right), \\
\frac{D a}{D t} & =\frac{\partial a}{\partial t}+J(\psi, a), \\
J(a, b) & =\frac{\partial a}{\partial x} \frac{\partial b}{\partial y}-\frac{\partial a}{\partial y} \frac{\partial b}{\partial x}
\end{aligned}
$$

Here, $\psi$ is the geostrophic streamfunction, $\nabla^{2}$ is the horizontal Laplacian, $D / D t$ is the advective derivative, $z \equiv-\ln \left(p / p_{1}\right)$ with $p_{1}$ a reference pressure level, $N$ is the Brunt-Väisälä frequency, $H=R T / g$ is the pressure scale height, $T$ is the temperature, $R$ is the gas constant, $g$ is the gravitational acceleration, and $f=f_{0}+\beta y$ is the Coriolis parameter.
We use a normal-mode expansion in the vertical coordinate, in which the streamfunction is written as

$$
\psi(x, y, z, t)=\sum_{n=0}^{N} \psi_{n}(x, y, t) \Phi_{n}(z),
$$

where the functions $\Phi_{n}(z)$ are the eigenfunctions of the vertical operator in (2.1), given by

$$
\mathscr{L} \Phi_{n}+\lambda_{n}^{2} \Phi_{n}=0,
$$

and $\lambda_{n}^{-1}$ is called the $n$th baroclinic deformation radius.

For a Jovian atmosphere, with a thin, stably stratified weather layer $\left(z_{1}<z<z_{2}\right)$ lying hydrostatically above a very deep, neutrally stratified, fluid interior $\left(z_{0}<z\right.$ $\left.<z_{1}\right)$ with $\left(z_{1}-z_{0}\right) \gg\left(z_{2}-z_{1}\right)$, the baroclinic $(n \geqslant 1)$ modes are confined to the weather layer and have $\Phi_{n}$ $=0$ for $z \leqslant z_{1}$, while the barotropic $\left(n=0, \lambda_{0}^{2}=0\right)$ mode has constant amplitude in both the weather layer and interior, with $\Phi_{0}=1$ for $z_{0}<z<z_{2}$ (Achterberg and Ingersoll 1989). A rigid lid $\left(d \Psi_{n} / d z=0\right)$ is assumed at $z_{2}$. Thus, the barotropic mode represents motions in the interior, while the baroclinic modes represent motions confined to the weather layer. The equation for the time evolution of mode $n$ is obtained by inserting (2.3) and (2.4) into (2.1), multiplying by $e^{-\left(z-z_{1}\right)} \Phi_{n}$, and integrating from $z_{0}$ to $z_{2}$. This gives

$$
\frac{\partial}{\partial t}\left(\nabla^{2} \psi_{0}\right)+\beta \frac{\partial \psi_{0}}{\partial x}+J\left(\psi_{0}, \nabla^{2} \psi_{0}\right)=0,
$$

for the barotropic mode, and

$$
\begin{aligned}
& \frac{\partial}{\partial t}\left(\nabla^{2} \psi_{n}-\lambda_{n}^{2} \psi_{n}\right)+\beta \frac{\partial \psi_{n}}{\partial x} \\
& +J\left(\psi_{0}, \nabla^{2} \psi_{n}-\lambda_{n}^{2} \psi_{n}\right)+J\left(\psi_{n}, \nabla^{2} \psi_{0}\right) \\
& \quad+\sum_{l, m=1}^{N} \gamma_{l m n} J\left(\psi_{l}, \nabla^{2} \psi_{m}-\lambda_{m}^{2} \psi_{m}\right)=0
\end{aligned}
$$

for the baroclinic modes, where the interaction coefficients $\gamma_{l m n}$ are given by

$$
\gamma_{l m n}=\int_{z_{1}}^{z_{2}} e^{-\left(z-z_{1}\right)} \Phi_{l} \Phi_{m} \Phi_{n} d z
$$

The quantity $q_{n} \equiv \nabla^{2} \psi_{n}-\lambda_{n}^{2} \psi_{n}$ is the potential vorticity of the $n$th mode. The potential vorticity at a given altitude, $q(z)$, is calculated by summing over the potential vorticity of each mode and adding in the planetary vorticity term $\beta y$ :

$$
q(x, y, z, t)=\beta y+\sum_{n=0}^{N}\left(\nabla^{2}-\lambda_{n}^{2}\right) \psi_{n}(x, y, t) \Phi_{n}(z),
$$

noting that $\lambda_{0}^{2}=0$. A detailed derivation of the modal model for a Jovian atmosphere with a deep, neutrally stratified interior is given by Achterberg and Ingersoll (1989). 
A major effect of the deep interior is that the barotropic mode is unaffected by the baroclinic modes (i.e., motions in the interior are not affected by the weather layer), although the baroclinic modes are affected by the barotropic mode. Because in this paper we are interested only in motions in the weather layer, we do not solve (2.5) but assume that motions in the interior are zonally symmetric such that $\psi_{0}=\psi_{0}(y)$, which is a solution to $(2.5)$. The barotropic mode then represents the effect of motions in the fluid interior upon motions in the weather layer.

An $N=1$ model is equivalent to the $11 / 2$-layer (reduced gravity), quasigeostrophic model that has often been used to study Jovian vortices (Achterberg and Ingersoll 1989). In this paper we use $N=2$, which allows for the possibility of baroclinic instability and for motions in the weather layer that vary with altitude. The calculation of the modes $\Phi_{n}(z)$, interaction coefficients $\gamma_{l m n}$, and ratio of second baroclinic deformation radius. to first baroclinic deformation radius $\lambda_{2}^{-1} / \lambda_{1}^{-1}$ are discussed in detail by Achterberg and Ingersoll (1989). We use their results for a molar mixing ratio for water of $10^{-3}$ and frozen equilibrium for the ortho-parahydrogen conversion. The structure of the modes is shown in Fig. 1, and the interaction coefficients $\gamma_{l m n}$ are shown in Table $I$.

The equations in this paper have been nondimensionalized by a length scale $L$ and velocity scale $U$, chosen such that model vortices have unit radius and

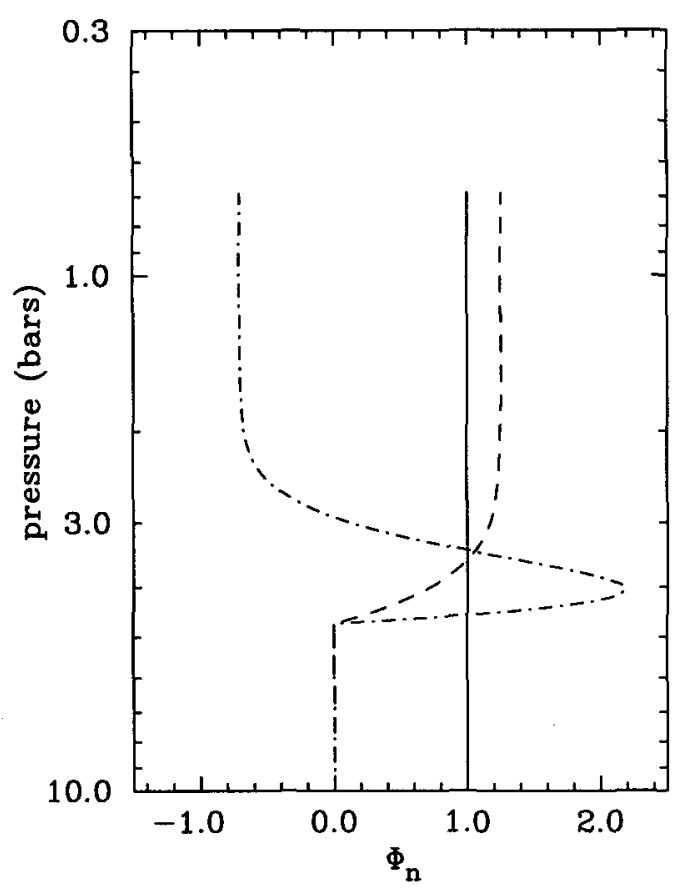

FIG. 1. Structure of the barotropic $(n=0$, solid line $)$ and first two baroclinic modes $\Phi_{n}(z)(n=1$, dashed line, and $n=2$, dot-dashed line).
TABLE 1. Parameters for normal-mode model of Jupiter.

$$
\begin{aligned}
\lambda_{2} / \lambda_{1} & =4.50 \\
\gamma_{111} & =1.176 \\
\lambda_{112} & =-0.1506 \\
\gamma_{122} & =0.8125 \\
\gamma_{222} & =1.365
\end{aligned}
$$

amplitude. The nondimensional variables can be related to dimensional variables through the relations $x_{d}$ $=L x, t_{d}=(L / U) t, \psi_{d}=L U \psi, \beta_{d}=\left(U / L^{2}\right) \beta$, and $\lambda_{d}$ $=\lambda / L$, where the dimensional variables are denoted by the $d$ subscript.

We solve (2.6) numerically using second-order centered finite differences, with the energy- and enstrophyconserving Jacobian of Arakawa (1966). The time integration uses leapfrog time steps, followed every 20 steps by a pair of leapfrog trapezoidal time steps, which damp the computational mode (Haltiner and Williams 1980). For long integrations (more than 8000 time steps), an eddy viscosity term of the form $\nu \nabla^{4} \psi_{n}$ is added to the right-hand side of (2.6), with $\nu=10^{-5}$. Repeating calculations with different values of $\nu$ showed no change in the qualitative behavior of the solutions. The domain is a channel with $0 \leqslant y \leqslant 3 \pi$; the width of the channel in $x$ is usually $0 \leqslant x \leqslant 3 \pi$, although $0 \leqslant x \leqslant 6 \pi$ is sometimes used. Most simulations were done on a $128 \times 128$ grid, which gives a resolution of $\delta x=0.074$. Some simulations were done on a $64 \times 64$ grid, and a few simulations were repeated on a $256 \times 256$ grid to examine the effects of the grid resolution. Boundary conditions are $\psi_{n}$ periodic in $x$, $d \psi_{n} / d x=\partial^{2} \bar{\psi}_{n} / \partial t \partial y=0$ at $y=0$ and $y=3 \pi$, where the overbar indicates a zonal average, which are equivalent to no normal flow at the boundary. In simulations with $\nu \neq 0$, the additional condition that the potential vorticity be constant and independent of time at the solid boundaries is used.

Equation (2.6) does not conserve the energy of the weather layer unless $\psi_{0}=0$, because the deep interior can act as a source or sink of energy. Conservation of energy by the numerical model is checked by comparing the rate of change of the energy of the model with the expected value calculated from the streamfunction; these usually agree to within a factor of a few times $10^{-4}$.

\section{Vortices on an $f$ plane with no zonal flow}

We begin by considering the nonlinear evolution of isolated vortices on an $f$ plane in the absence of a mean zonal flow $\left(\psi_{0}=0 ; \psi_{1} \rightarrow 0\right.$ and $\psi_{2} \rightarrow 0$ far from the initial vortex). To examine the nonlinear stability of isolated $f$-plane vortices in our Jovian model, we follow the time evolution of a nearly circular vortex using the numerical methods described in section 2 . The initial condition is a Gaussian streamfunction of unit radius 
with an elliptical perturbation (to excite any instability):

$$
\begin{aligned}
\psi_{n}=A_{n} \exp \left[-\left(\frac{x}{1.05}\right)^{2}-\left(\frac{y}{0.95}\right)^{2}\right] & \text { for } n=1,2
\end{aligned}
$$

with $\psi_{0}=0$. With this initial condition there are two independent parameters: $\lambda_{1}$, the size of the initial vortex divided by the first internal deformation radius, and $s$ $\equiv A_{2} / A_{1}$, the ratio of the amplitude of the second baroclinic mode to the amplitude of the first baroclinic mode of the initial vortex. Thus, $s=0$ represents an equivalent barotropic vortex. Increasing the magnitude of $s$ increases the baroclinicity of the vortex. Vortices with $-0.214 \leqslant s \leqslant 1.769$ have the same sign of vorticity at all altitudes. If $s \approx-0.214$, the contributions from the first and second baroclinic modes nearly cancel below $\sim 4$ bar because then $A_{1} d \Phi_{1} / d z \approx-A_{2} d \Phi_{2} /$ $d z$ (see Fig. 1 ) at these altitudes. If $s \approx 1.769$, the contributions from the first and second baroclinic modes cancel at altitudes above $\sim 2$ bar because $A_{1} \Phi_{1}$ $\approx-A_{2} \Phi_{2}$ at these altitudes. For large $|s|$, the vertical structure of the vortex resembles the second baroclinic mode, while for small $|s|$ the vertical structure of the vortex resembles the first baroclinic mode. The actual mode amplitudes are determined by requiring that $A_{1}^{2}$ $+A_{2}^{2}=1$ and that $A_{1}>0$. Changing the signs of $A_{1}$ and $A_{2}$ merely changes the direction of rotation of the vortex but does not affect the dynamics in the quasigeostrophic limit. Numerical simulations were performed for $0.01 \leqslant \lambda_{1}^{2} \leqslant 3.0$ and $-2 \leqslant s \leqslant 2$. This covers vortices with diameters ranging from one-fifth of the first internal deformation radius up to about four times the first internal deformation radius. The deformation radius on Jupiter is unknown but has been estimated to be in the range of $500-5000 \mathrm{~km}$, with the most likely value on the order of $1000 \mathrm{~km}$ (Achterberg and Ingersoll 1989). Thus, our simulations cover vortices ranging in size from the smallest observed vortices up to a few thousand kilometers. Our simulations do not reach the size of the largest observed vortices (the GRS and White Ovals); using values of $\lambda_{1}^{2} \geqslant 3$ in our models is impractical, as it requires a prohibitively small grid spacing to adequately resolve the deformation radius of the second baroclinic mode.

We find four different behaviors, depending upon the values of $s$ and $\lambda_{1}^{2}$ : horizontal fragmentation of the initial vortex, usually into two dipoles; elongation of the vortex followed by a decrease in eccentricity and generation of a tripolar structure, which is very similar to the behavior of the rapidly/slowly deformed ring of Ikeda (1981); vertical breakup of the vortex into separate vortices at different altitudes, an example of the internal barotropic instability discussed by Gent and McWilliams (1986); and long-term stability in which the initial vortex persists for tens of rotations with little change. Each of these is discussed in more detail below.

\section{a. Horizontal fragmentation}

Figure 2 shows the time evolution of the streamfunction and potential vorticity for $\lambda_{1}^{2}=1.0$ and $s$ $=-2.0$, in which the sign of the vorticity changes with altitude. The streamfunction and potential vorticity have been evaluated at two pressure levels: $690 \mathrm{mb}$, corresponding roughly to the altitude of the visible clouds on Jupiter, and $3962 \mathrm{mb}$, corresponding to the maximum of the second-mode eigenfunction. We will refer to the streamfunction evaluated at $690 \mathrm{mb}$ as the "upper-layer streamfunction" and the streamfunction evaluated at $3962 \mathrm{mb}$ as the "lower-layer streamfunction." The streamfunction (and potential vorticity) at these altitudes displays qualitatively similar behavior to the streamfunction and potential vorticity in the upper and lower layers of a two-layer model. The vortex rapidly elongates, with the upper part of the vortex rotated with respect to the lower part, and pinches off in the center. This results in two dipolar structures, each with a vortex in the upper layer paired with a vortex of opposite-signed vorticity in the lower layer. The dipolar structures then move away from each other. The two vortices in each layer remain connected by filaments of potential vorticity that stretch out and become thinner as the vortices separate. This breakup into dipoles requires approximately one rotation time of the initial vortex. The breakup is accompanied by a $30 \%$ decrease in the potential energy of the second baroclinic mode between times $t=4$ and $t=9$, with a corresponding increase in the kinetic energy of the first baroclinic mode; this indicates that the energy source for the instability is baroclinic. Splitting of the initial vortex into multiple dipoles is observed only when the sign of the vorticity of the original vortex varies with altitude and when $\lambda_{1}^{2} \geqslant 0.3$ (or equivalently, when the diameter of the vortex is roughly greater than the first internal deformation radius), although not for all vortices that meet these conditions.

\section{b. Tripole formation}

Figure 3 shows the evolution of the streamfunction and potential vorticity for the case $\lambda_{1}^{2}=1.0$ and $s$ $=-0.5$, which also has oppositely signed vorticity in the upper and lower layers but with more energy in the first baroclinic mode than the previous case shown in Fig. 2. Initially the upper-layer part of the vortex becomes elongated, while the lower-layer part of the vortex splits into two smaller vortices. The upper-layer vortex then becomes more circular while another vortex with the same sign of vorticity as the upper-layer vortex forms below the upper-layer vortex and between the two lower-layer vortices. This forms a tripolar structure, with a central vortex flanked on either side by two vortices that have the opposite sign of vorticity as the central vortex. This tripolar structure then rotates as a unit with little change in structure. As with hori- 

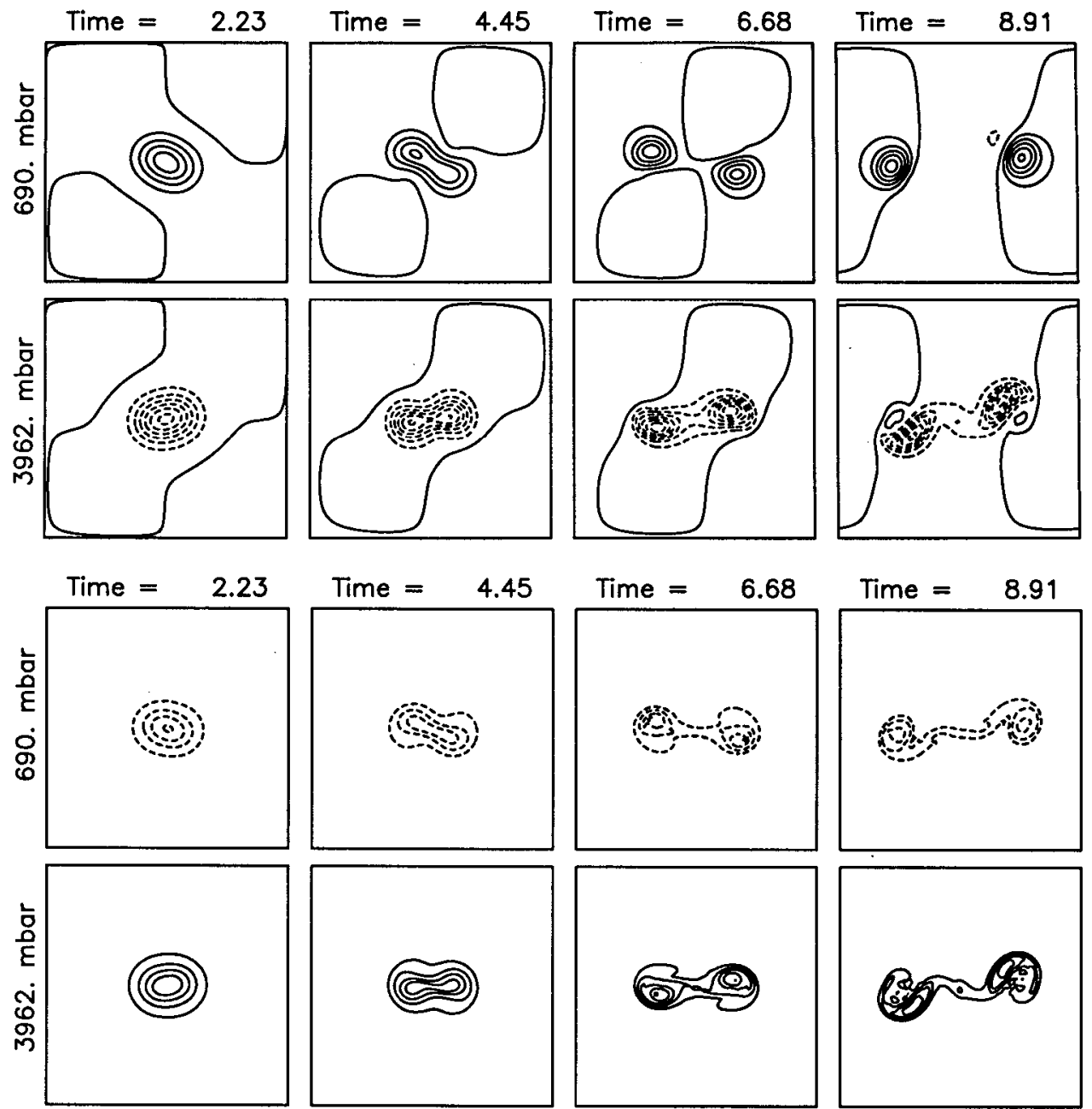

FIG. 2. Example of horizontal fragmentation. Time evolution of an $f$-plane vortex with $s=-2.0$ and $\lambda_{1}^{2}$ $=1.0$ at times $2.23,4.45,6.68$, and 8.91. (a) Streamfunction at $690 \mathrm{mb}$ (top row) and at $3962 \mathrm{mb}$ (bottom row). The contour interval is 0.25 , and negative contours are dashed. (b) Potential vorticity at $690 \mathrm{mb}$ with a contour interval of 5.0 (top row) and at $3962 \mathrm{mb}$ with a contour interval of 10.0 (bottom row). Negative contours are dashed, and the contours straddle 0 . In this figure and subsequent figures showing the streamfunction and potential vorticity contours, the horizontal coordinates range from 0 to $3 \pi$ in units of $L$, the initial vortex radius.

zontal fragmentation, the formation of the tripole is accompanied by a $50 \%$ decrease in the potential energy of the second baroclinic mode between times $t$ $=4$ and $t=16$ and a corresponding increase in the kinetic energy of the first baroclinic mode, indicating that tripole formation is a form of baroclinic instability.

Similar behavior is seen for some vortices with the same sign of vorticity at all altitudes but with a change in sign with altitude of the potential vorticity, as shown in Fig. 4 for $s=1.5$ and $\lambda_{1}^{2}=1.0$. In this case, the upper-layer vortex becomes stronger and more elongated and develops a pair of vortices with vorticity of the opposite sign of the original vortex on either side of it. As the side vortices develop, the lower-layer vortex elongates and then returns to a more circular shape. The result is again a tripolar structure.

The relative sizes and vertical structures of the central and flanking vortices depend upon the values of $s$ and $\lambda_{1}$. Decreasing the size or baroclinicity of the initial vortex results in smaller flanking vortices relative to the central vortex and central vortices with vertical structures closer to the vertical structure of the initial vortex. As with horizontal fragmentation, formation of tripoles occurs only when $\lambda_{1}^{2} \geqslant 0.3$ and only when the sign of the potential vorticity of the initial vortex varies with altitude. 

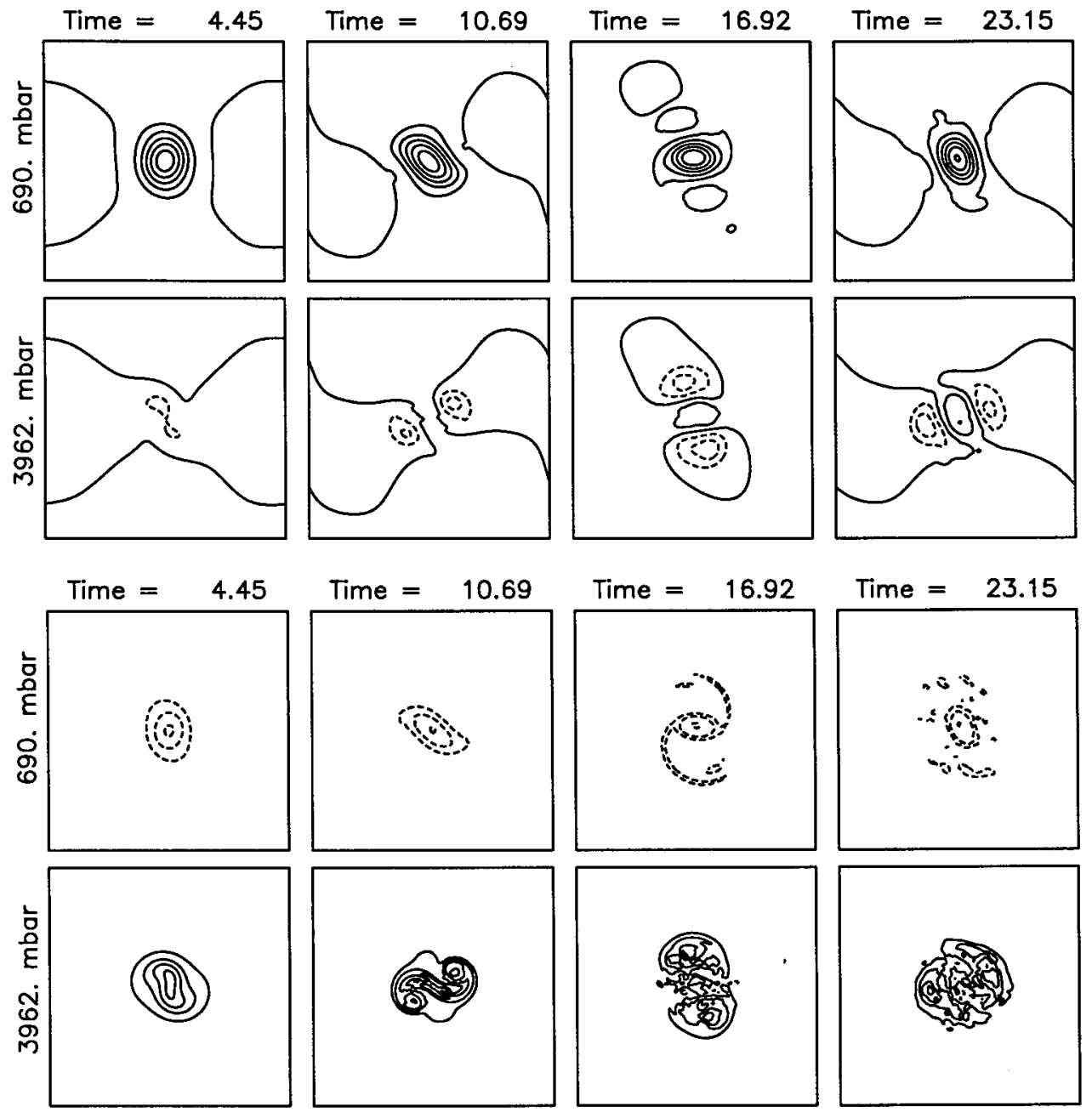

FIG. 3. Example of tripole formation. Time evolution of an $f$-plane vortex with $s=-0.5$ and $\lambda_{1}^{2}=1.0$ at times $4.45,10.69,16.92$, and 23.15. (a) Streamfunction at $690 \mathrm{mb}$ (top row) and at $3962 \mathrm{mb}$ (bottom row). The contour interval is 0.25 , and negative contours are dashed. (b) Potential vorticity at $690 \mathrm{mb}$ (top row) and at $3962 \mathrm{mb}$ (bottom row). The contour interval is 5.0, negative contours are dashed, and the contours straddle 0.

\section{c. Vertical fragmentation}

Figure 5 shows the evolution of the streamfunction for $s=0.5$ and $\lambda_{1}^{2}=0.03$. The vertical axis of the vortex becomes tilted, and the upper- and lower-layer parts of the vortex separate and slowly move away from each other in opposite directions, leaving two vortices of limited vertical extent. Splitting of the vortex is accompanied by a $50 \%$ decrease in the kinetic energy of the first baroclinic mode between times $t=30$ and $t=60$ and an increase in both the kinetic and potential energies of the second baroclinic mode, indicating that the instability is barotropic. This behavior is only observed when $\lambda_{1}^{2} \leqslant 0.1$ and the vortex has the same sign of vorticity in both layers. Under these conditions, vertical fragmentation can occur for equivalent barotropic vortices $(s=0)$. These vortices are representable in the
$N=1$ model, and therefore in the common equivalent barotropic models, and are stable in such models. The time scale for vertical fragmentation is much longer than for horizontal fragmentation, requiring on the order of 10 vortex rotation times.

\section{d. Discussion}

Figure 6 shows the regions of $\lambda_{1}^{2}-s$ parameter space in which each of the aforementioned behaviors occurs. Horizontal fragmentation and tripole formation occur only for vortices with diameters roughly equal to or larger than the first baroclinic deformation radius and with a second-mode strong enough that the sign of the potential vorticity changes with altitude. Horizontal fragmentation is further confined to vorticies in which the sign of the vorticity changes with altitude. Vertical 

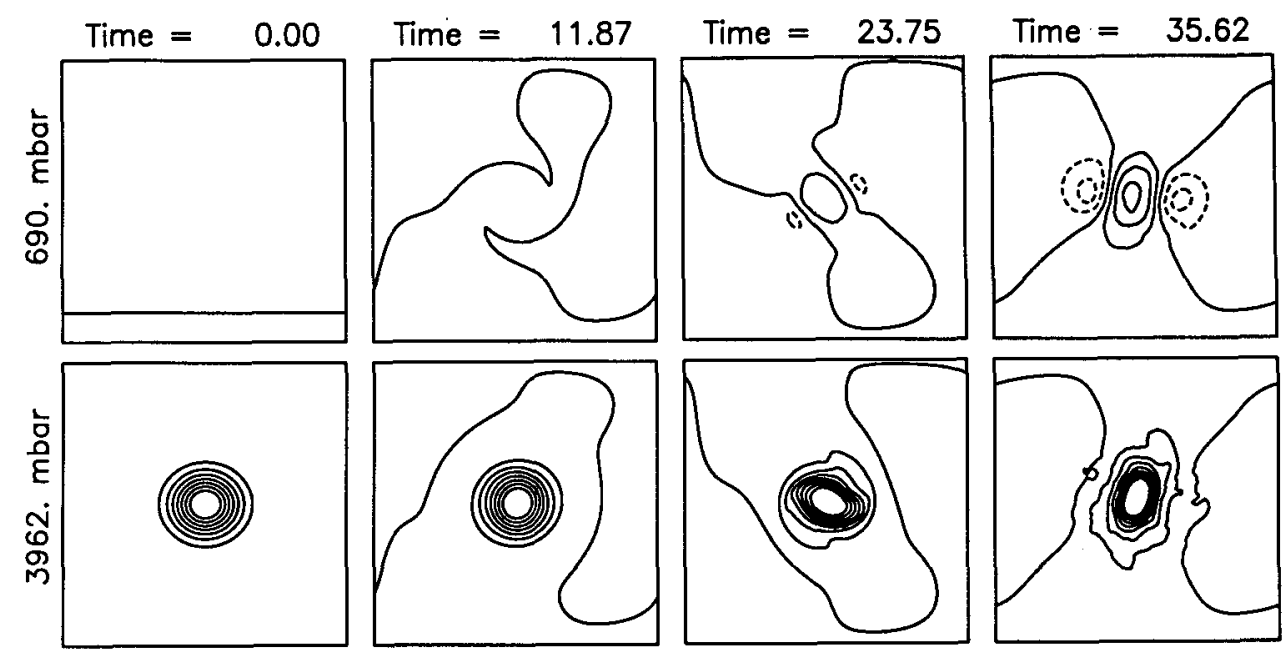

Fig. 4. Example of tripole formation for a vortex with the same sign of vorticity at all altitudes. Time evolution of an $f$-plane vortex with $s=1.5$ and $\lambda_{1}^{2}=1.0$ at times $0.0,12.87,23.75$, and 35.62. The top row shows the streamfunction evaluated at $690 \mathrm{mb}$, and the bottom row shows the streamfunction evaluated at $3962 \mathrm{mb}$. The contour interval is 0.25 and negative contours are dashed.

fragmentation occurs only for vortices with diameters smaller than the first baroclinic deformation radius and with the same sign of vorticity at all altitudes. Stability occurs for vortices with diameters larger than the radius of deformation that are barotropic or nearly barotropic and for vortices smaller than the radius of deformation with vorticity that changes sign with altitude.

These results are qualitatively similar to results from two-layer models with a solid lower boundary, although there are definite quantitative differences. Flierl (1988) examined the linear instability of vortices in a two-mode contour dynamics model with a solid lower boundary. He found that vortices with a strong enough baroclinic component (equivalent to $|s| \geqslant 0.5$ ) are baroclinically unstable if their radius is larger than about twice the deformation radius. The exact stability boundaries are dependent upon the assumed vertical stratification. The minimum value of $|s|$ for which instability occurs decreases as $\lambda_{1}^{2}$ is increased. Flierl also found that vortices with the same sign of vorticity at all altitudes and a radius smaller than about $11 / 2$ times the radius of deformation are subject to an instability that causes a twisting and tilting of the vertical axis of the vortex. This matches our results that baroclinic instability occurs for large enough vortices with a strong enough baroclinic component, that barotropic instabil-

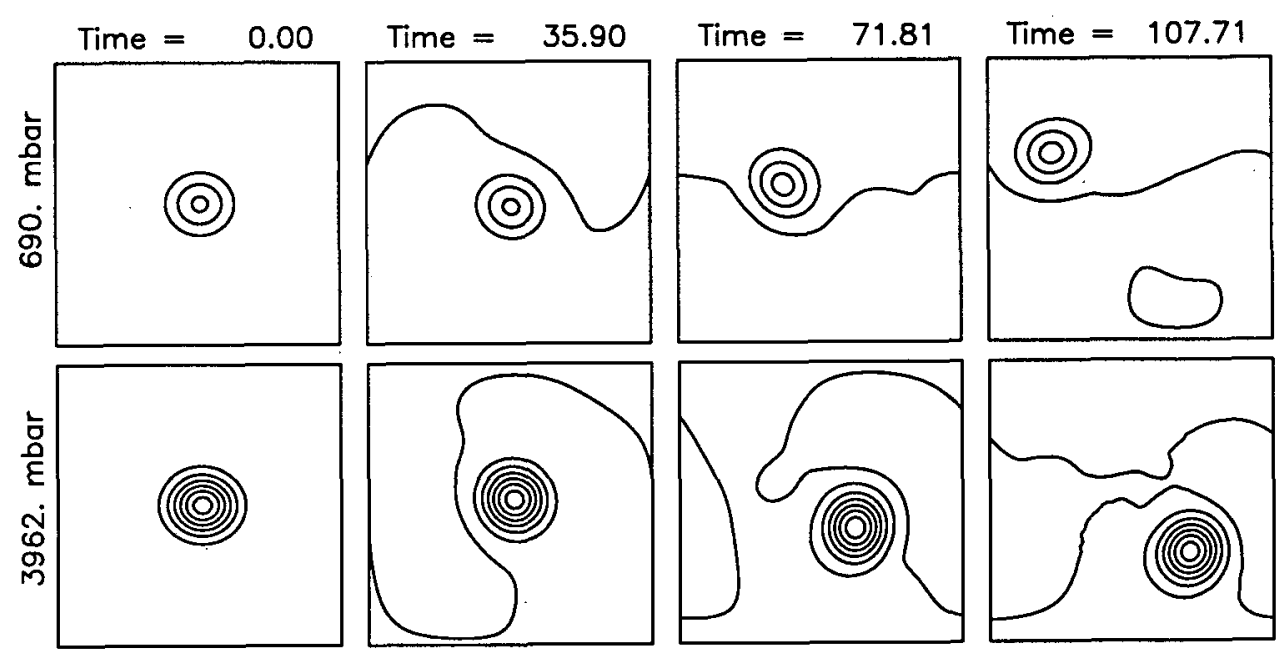

FIG. 5. Example of vertical fragmentation. Time evolution of an $f$-plane vortex with $s=0.5$ and $\lambda_{1}^{2}=0.03$ at times $0.0,35.90,71.81$, and 107.71 . The top row shows the streamfunction evaluated at $690 \mathrm{mb}$, and the bottom row shows the streamfunction evaluated at $3962 \mathrm{mb}$. The contour interval is 0.25 . 


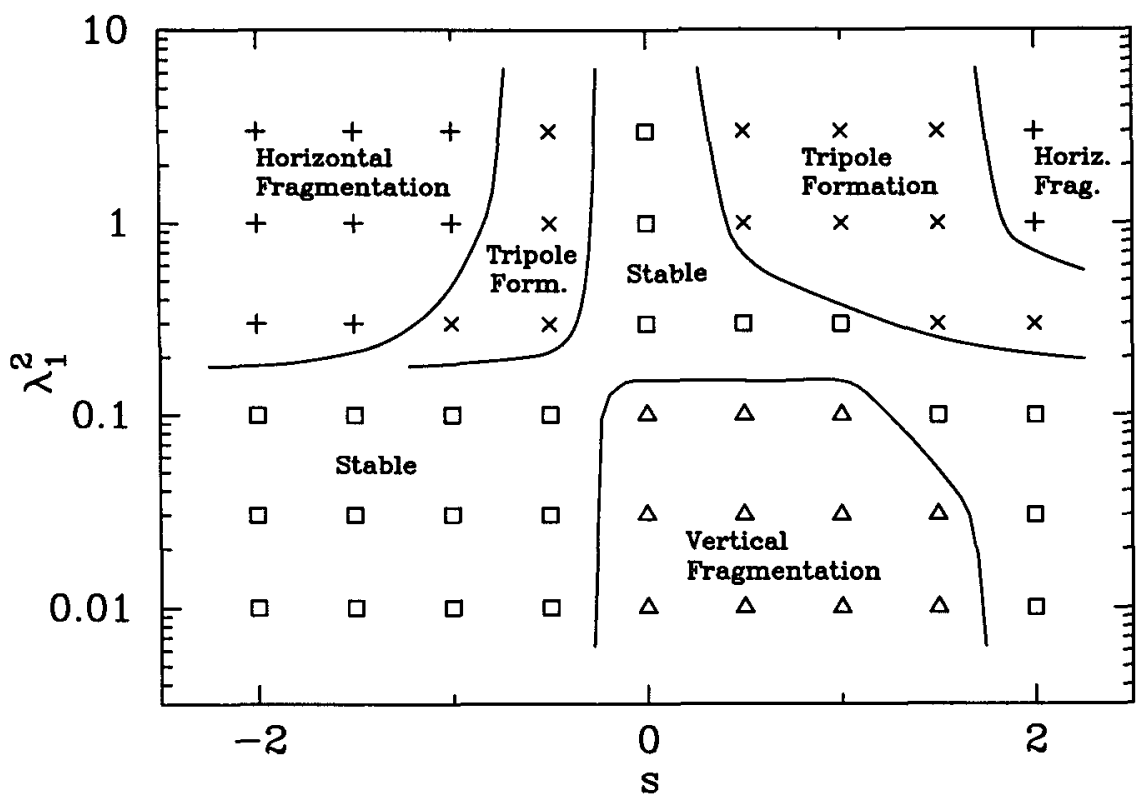

FIG. 6. Regime diagram for the behavior of vortices on the $f$ plane with no mean zonal flow. The horizontal axis is $s$, the ratio of second baroclinic mode amplitude to first baroclinic mode amplitude for the initial vortex. The vertical axis is $\lambda_{1}^{2}$, the square of the radius of the vortex relative to the first internal radius of deformation. The open squares represent stable vortices (those that survive to at least time 400 without becoming unstable), the plus signs represent horizontal fragmentation (baroclinic instability), the crosses represent tripole formation, and the open triangles represent vertical fragmentation (internal barotropic instability).

ity occurs for small enough vortices with the same sign of vorticity at all altitudes, and that large barotropic vortices are stable. The major difference is that our stability boundaries occur for a value of $\lambda_{1}$ a factor of about 4-5 times smaller than found by Flierl.

Our results may also be compared to models of Ikeda (1981) and Gent and McWilliams (1986). Ikeda (1981) considered the stability of Gaussian vortices in a two-layer, quasigeostrophic model with solid lower boundary, considering only vortices that were strongest in the thinner layer (equivalent to $1.0 \leqslant s \leqslant 1.7$ in our model). For a vortex confined to the thinner layer (equivalent to $s \approx 1.7$ in our model), Ikeda found that baroclinic instability occurs for vortices with radii larger than about twice the deformation radius. His nonlinear calculations show tripole formation occurring for vortices larger than twice the deformation radius, changing to horizontal fragmentation for vortices larger than about $31 / 2$ times the deformation radius. The splitting of a baroclinically unstable vortex into multiple dipolar structures was also seen in nonlinear twolayer contour dynamics simulations by Helfrich and Send (1988).

Gent and McWilliams (1986) considered the stability of barotropic vortices ( $s=0$ in our model), allowing the perturbations to be baroclinic. They found that the most unstable mode for barotropic Gaussian vortices has nontrivial altitude-dependent vertical structure.
Their nonlinear numerical simulations using a twolayer model show that this instability, which they call "internal barotropic instability," resulted in the vertical fragmentation of the vortex. Their linear calculations show that for a Gaussian vortex this instability occurs for $\lambda_{1}^{2}<1.6$.

In all the preceding cases discussed we find the stability boundaries at values of $\lambda_{1}$ that are about a factor of 5 lower than found by other authors using models with a solid lower boundary. This factor of 5 is similar to the ratio of 4.5 between the second and first baroclinic deformation radii in our model, which suggests that the second baroclinic deformation radius may be the important factor in determining the stability boundaries. This would not be surprising since in our model the second baroclinic mode is needed to produce baroclinic instability. This conjecture may be tested by varying $\lambda_{1}^{2}$ and $\lambda_{2}^{2}$ separately instead of keeping the ratio $\lambda_{2} / \lambda_{1}$ constant. Using $s=0$, we find that vertical fragmentation is dependent primarily on the value of $\lambda_{2}^{2}$, although increasing $\lambda_{1}^{2}$ decreases the growth rate of the instability slightly. Results with $s=-1.0$ and $s=1.0$ are quite different; increasing $\lambda_{1}^{2}$ while keeping $\lambda_{2}^{2}$ constant has the opposite effect of increasing $\lambda_{2}^{2}$ while keeping $\lambda_{1}^{2}$ constant. For example, with $s=-1.0$ and $\lambda_{1}^{2}=0.1$, we find stable vortices for $\lambda_{2}^{2}=0.6$ and $\lambda_{2}^{2}$ $=2.0$, and horizontal fragmentation for $\lambda_{2}^{2}=6.0$ and $\lambda_{2}^{2}=20$. With $s=-1.0$ and $\lambda_{2}^{2}=6.0$, we find horizontal 


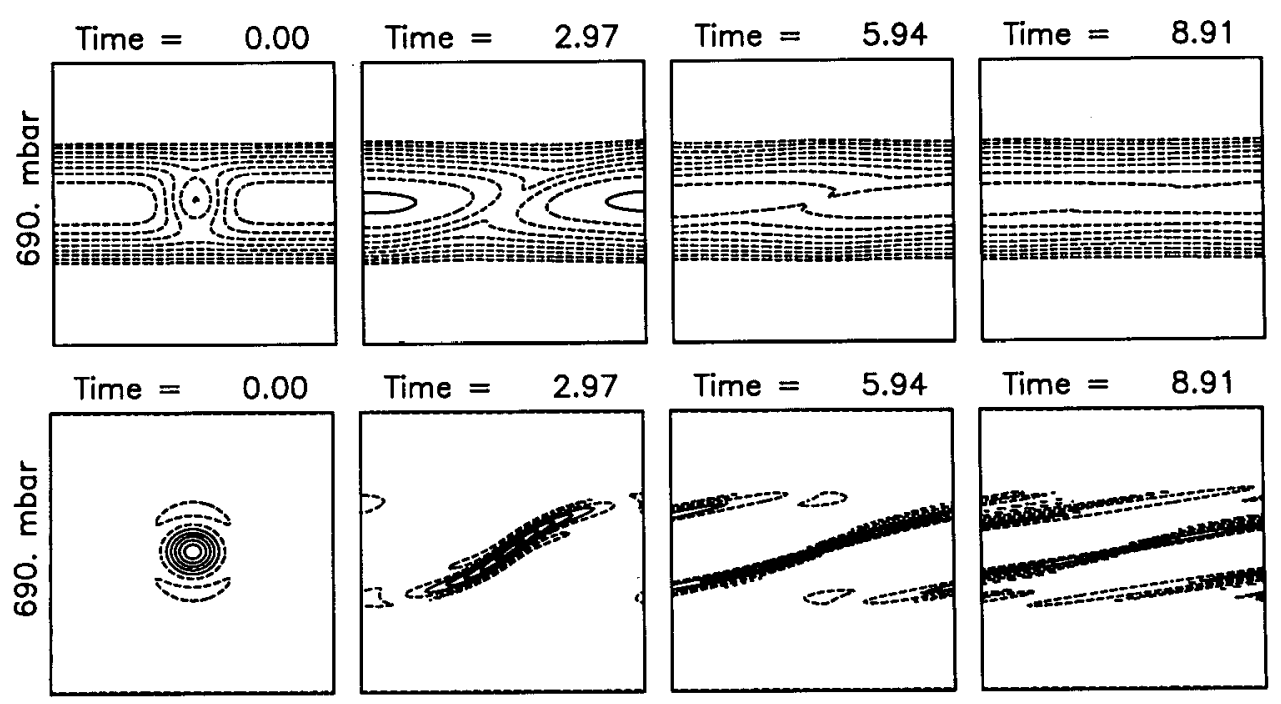

Fig. 7. Example of a vortex rotating counter to the mean zonal flow. Time evolution of an $f$-plane vortex with $s=0.0, \lambda_{1}^{2}=1.0$, and $U_{0}=1.0$ at times $0.0,2.97,5.94$, and 8.91 . (a) Streamfunction at $690 \mathrm{mb}$. The contour interval is 0.25 , and negative contours are dashed. Contours for $\psi<-2.0$ are not shown. (b) Potential vorticity at $690 \mathrm{mb}$. The contour interval is $\mathbf{1 . 0}$ and negative contours are dashed.

fragmentation for $\lambda_{1}^{2}=0.03$ and $\lambda_{1}^{2}=0.1$, tripole formation for $\lambda_{1}^{2}=0.3$, and stable vortices for $\lambda_{1}^{2}=1.0$ and $\lambda_{1}^{2}=3.0$. It thus appears that for the forms of baroclinic instability, increasing $\lambda_{1}^{2}$ while holding $\lambda_{2}^{2}$ constant stabilizes the vortex. The effect of using a deep fluid layer instead of a solid lower boundary can then be understood by noting that using a solid lower boundary is equivalent to setting $\lambda_{1}^{2}=0.0$ in the calculations described above (i.e., with a solid lower layer, the barotropic and first baroclinic mode are used instead of the first two baroclinic modes used in the presence of the deep lower layer). Thus, we see that the use of the fluid lower boundary acts to stabilize the flow in the weather layer, particularly against barotropic instability. A similar effect of a fluid lower boundary reducing the growth rates of baroclinic instability of a mean zonal flow has been found by Gierasch et al. (1979) and Conrath et al. (1981).

\section{Effects of a barotropic mean zonal flow}

We now consider the effects of a barotropic mean zonal flow on the solutions of the previous section. To do this, a.barotropic mean zonal flow $\psi_{0}=-\frac{1}{2} U_{0} y^{2}$ with constant clockwise shear is added to the barotropic mode. The case of a baroclinic mean zonal flow is not considered. This barotropic mean zonal flow has no meridional potential vorticity gradient and thus will not support Rossby waves. Although such a zonal flow is not realistic for Jupiter, it allows us to examine the effects of a mean zonal flow upon the stability of vortices without having to separate the effects of the shear from the effects of interactions with Rossby waves. The effects of a mean zonal potential vorticity gradient will be discussed in the next section. Superimposed upon the zonal flow is a Gaussian vortex as described in the previous section. We now have three independent parameters, $\lambda_{1}, s$, and $U_{0}$, representing, respectively, the size of the vortex relative to the deformation radius, the vertical structure of the vortex, and the vorticity of the shear flow. The effects of the shear on the results of the previous section are discussed herein.

\section{a. Effects of zonal flows on stable $f$-plane vortices}

When shear is added, the behavior of a vortex that is stable on the $f$ plane depends upon the sign of the vorticity of the zonal flow. If the vorticity of the vortex has the opposite sign from the vorticity of the background shear flow, the vortex becomes sheared out by the mean flow and is destroyed (Fig. 7). This is similar to the behavior in a nonrotating single-layer system of an elliptical patch of constant vorticity embedded in a background flow of constant shear with the opposite sign of vorticity (Kida 1981; Meacham et al. 1990). If the initial vortex has a different sign of vorticity at different altitudes, the portion of the vortex rotating counter to the flow is stretched out and destroyed, leaving behind a vortex of limited vertical extent (Fig. 8).

A vortex with the same sign of vorticity as the mean flow at all altitudes and that is stable in the absence of the mean flow is also stable in the presence of the mean flow. However, the vortex is not, in general, steady; the aspect ratio and orientation oscillate quasi-periodically with slight variations in amplitude and period. As an example, for a vortex with $s=0.0, \lambda_{1}^{2}=1.0$, and $U_{0}$ $=0.5$, the aspect ratio (ratio of semimajor to semiminor axes) oscillates between 1.2 and 1.6 and the angle be- 

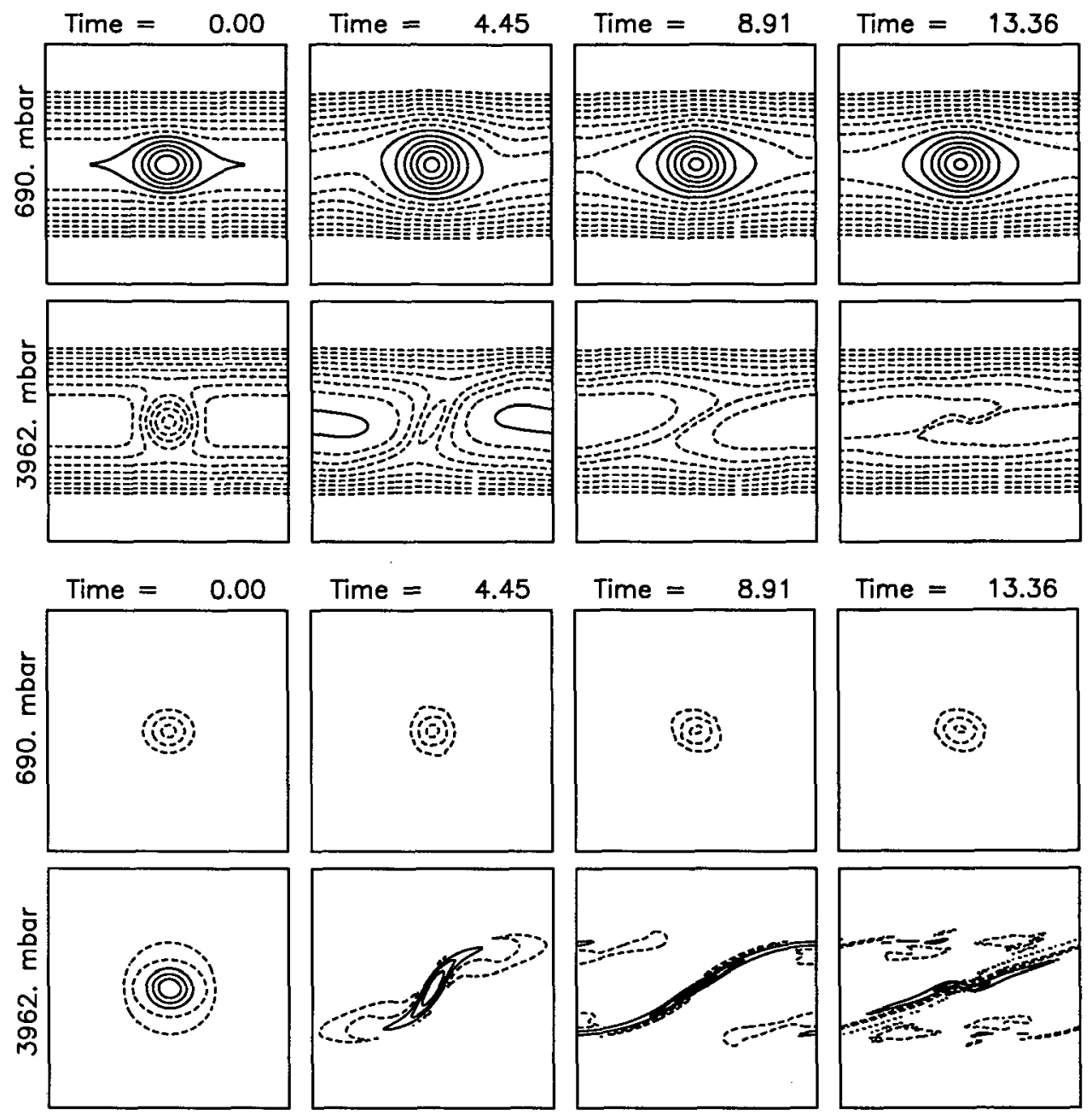

FIG. 8. Example of a vortex rotating counter to the mean zonal flow at some altitudes. Time evolution of an $f$-plane vortex with $s=-2.0, \lambda_{1}^{2}=0.03$, and $U_{0}=0.5$ at times $0.0,4.45,8.91$, and 13.36. (a) Streamfunction at $690 \mathrm{mb}$ (top row) and at $3962 \mathrm{mb}$ (bottom row). The contour interval is 0.25 and negative contours are dashed. (b) Potential vorticity at $690 \mathrm{mb}$ (top row) and at $3962 \mathrm{mb}$ (bottom row). The contour interval is 1.0, negative contours are dashed, and the contours straddle 0.

tween the semimajor axis, and the direction of the mean zonal flow oscillates between $\pm 20^{\circ}$ with a period of about 20 (roughly three times the vortex rotation period). The oscillations in aspect ratio and orientation differ in phase by approximately $90 \mathrm{deg}$, such that the aspect ratio is increasing while the semimajor axis is tilted counterclockwise (i.e., in the direction opposite to the rotation of the vortex from the direction of the mean zonal flow. These oscillations are similar to those seen in a single-layer contour dynamics model (Kida 1981).

The oscillations in shape and orientation are accompanied by transfers of energy between the vortex and the mean zonal flow, which also show quasi-periodic oscillations. The main energy conversion term is the conversion of mean kinetic energy to eddy kinetic energy $\left\{\bar{K} \cdot K^{\prime}\right\}$, resulting in variation of the kinetic en- ergy of the vortex of about 5\%. The oscillations of $\left\{\bar{K} \cdot K^{\prime}\right\}$ are approximately 90 degrees out of phase with the oscillations of aspect ratio and roughly in phase with the oscillations of the orientation, such that the mean flow is adding energy to the vortex when the aspect ratio of the vortex is decreasing and the vortex is tilted clockwise from alignment of the major axis with the zonal flow. The oscillations persist throughout the computation, with no sign of decaying.

\section{b. Effects of zonal flows on horizontal fragmentation}

The effect of a zonal flow on horizontal fragmentation is shown in Fig. 9 for $s=-2.0, \lambda_{1}^{2}=1.0$, and $U_{0}$ $=0.5$. These are the parameters used in Fig. 2, with the addition of a mean zonal flow. The initial behavior of the vortex is similar to the behavior without the zonal 

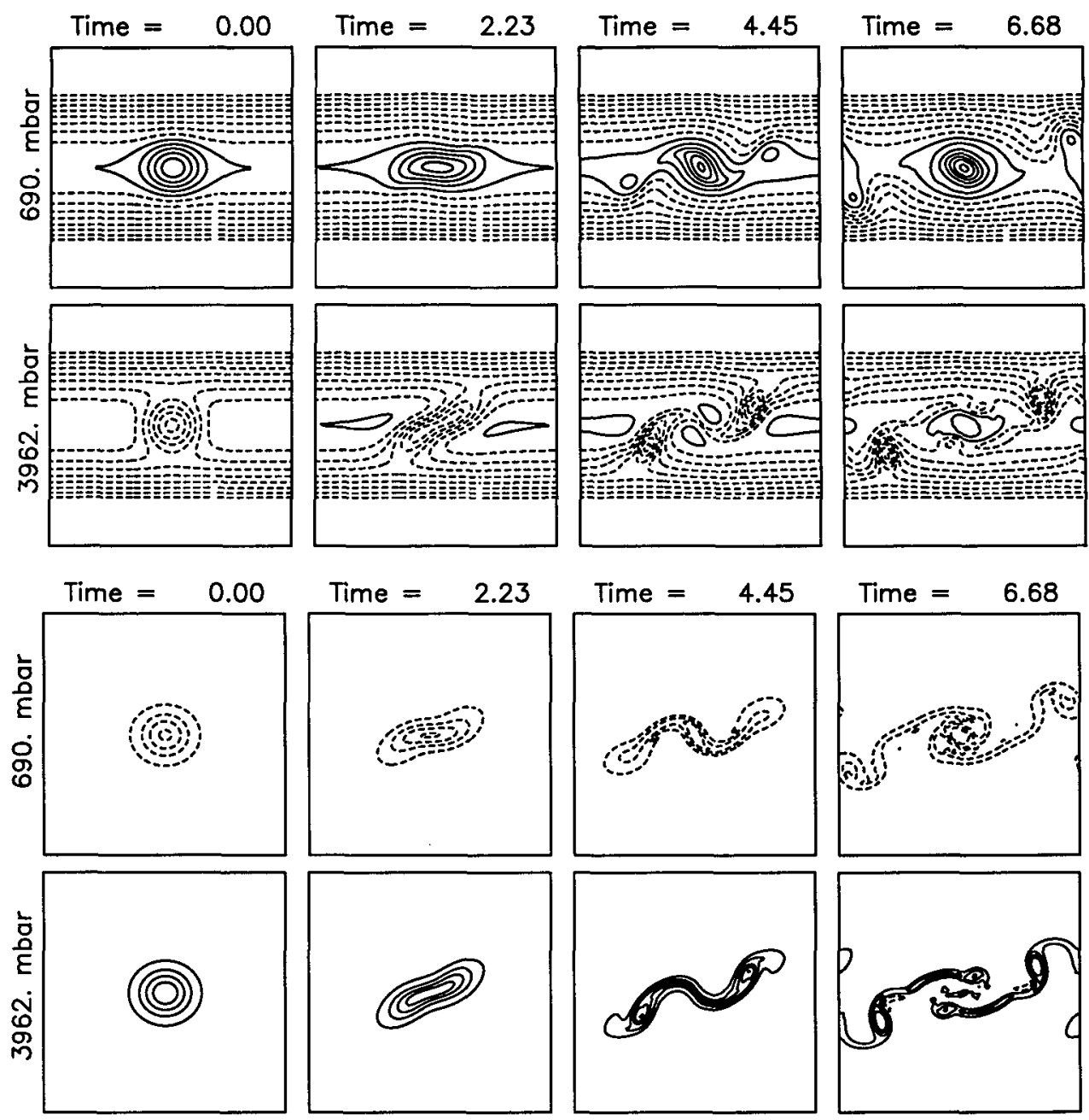

Fig. 9. Effect of mean zonal shear on horizontal fragmentation. Time evolution of an $f$-plane vortex with $s=-2.0, \lambda_{1}^{2}=1.0$, and $U_{0}=0.5$ at times $0.0,2.23,4.45$, and 6.68 . (a) Streamfunction at $690 \mathrm{mb}$ (top row) and at $3962 \mathrm{mb}$ (bottom row). The contour interval is 0.25 and negative contours are dashed. (b) Potential vorticity at $690 \mathrm{mb}$ with a contour interval of 5.0 (top row) and at $3962 \mathrm{mb}$ with a contour interval of $\mathbf{1 0 . 0}$ (bottom row). Negative contours are dashed, and the contours straddle 0.

flow: the vortices become elongated with the upper and lower vortices tilted with respect to each other. In contrast to the case without the zonal flow, however, the upper-layer vortex (which has the same sign of vorticity as the zonal flow) does not split into two vortices. Instead it sheds two large filaments of potential vorticity with weak vortices at the ends, which become drawn out by the mean zonal flow. The lower-layer vortex still splits into two vortices, which are advected away from the main upper-layer vortex by the mean zonal flow. The result is that the original vortex is strongly modified, becoming smaller with a stable vertical structure (i.e., the resulting vortex is in a stable region of parameter space) but is not completely destroyed. As with horizontal fragmentation in the absence of the mean zonal flow, the primary energy conversion is from potential to kinetic energy, which indicates that the insta- bility is baroclinic. There are also significant contributions from the interactions with the mean zonal flow. The time scale for the adjustment and shedding of vortices is on the order of the turnaround time for the vortex but slightly faster than the time scale for splitting of the vortex in the absence of the zonal flow.

\section{c. Effects of zonal flows on tripole formation}

The effect of a zonal flow on tripole formation is shown in Fig. 10 for $s=-0.5, \lambda_{1}^{2}=1.0$, and $U_{0}=0.5$, which is the same as shown in Fig. 3 with the addition of a mean zonal flow. The upper-layer vortex becomes elongated and then returns to a more circular form, while in the lower layer a vortex spins up beneath the upper-layer vortex. The behavior is similar to the behavior without the vertical shear except that the lower- 

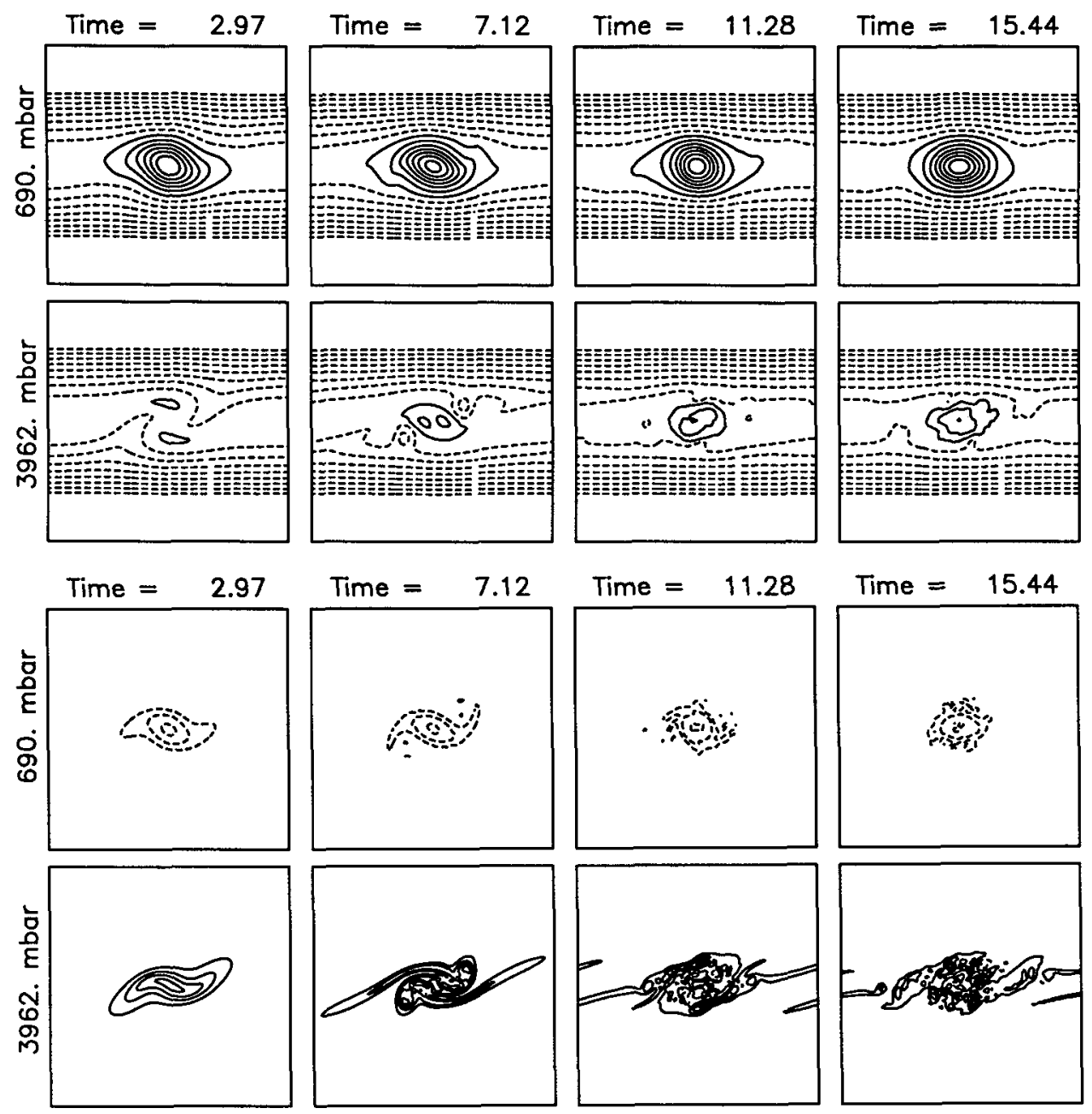

FIG. 10. Effect of mean zonal shear on tripole formation. Time evolution of an $f$-plane vortex with $s$ $=-0.5, \lambda_{1}^{2}=1.0$, and $U_{0}=0.5$ at times 2.97, 7.12, 11.28, and 15.44. (a) Streamfunction at $690 \mathrm{mb}$ (top row) and at $3962 \mathrm{mb}$ (bottom row). The contour interval is 0.25 and negative contours are dashed. (b) Potential vorticity at $690 \mathrm{mb}$ (top row) and at $3962 \mathrm{mb}$ (bottom row). The contour interval is 5.0, negative contours are dashed, and the contours straddle 0.

layer flanking vortices are inhibited by the mean zonal flow. The lower-layer potential vorticity patches, which would become the flanking vortices in the absence of the mean zonal flow, are instead sheared out by the zonal flow. The original vortex maintains its identity but the vertical structure adjusts to give a stable vortex. The energy conversion terms are dominated by quasiperiodic oscillations of $\left\{\bar{K} \cdot K^{\prime}\right\}$, as with the stable vortex in a mean zonal flow, although there is also a conversion of potential energy to kinetic energy during the first two vortex rotation times. The time scale for this adjustment is similar to the time scale for tripole formation in the absence of the mean zonal flow.

\section{d. Effects of zonal flows on vertical fragmentation}

In the case of vertical fragmentation by internal barotropic instability, the presence of a mean zonal flow greatly decreases the rate at which the upper and lower vortices separate from each other. As a result, the upper and lower vortices follow an ellipsoidal spiral trajectory that slowly expands. Figure 11 shows the trajectories of the maxima of the upper- and lower-layer streamfunctions for $s=0.5, \lambda_{1}^{2}=0.03$, and $U_{0}=0.5$. The axes show distance to the east and north, respectively, scaled by the radius $L$ of the initial vortex. The spiral trajectory results in oscillations of the latitude and longitude of the vortex, with a $90 \mathrm{deg}$ phase difference between the latitudinal and longitudinal oscillations. The longitude oscillation has a larger amplitude than the latitude oscillation, and the weaker of the upper- and lower-layer vortices has a larger oscillation amplitude than the stronger vortex. The amplitude and period of the oscillations increase with time. When the peak-to-peak amplitude of the latitude oscillations 


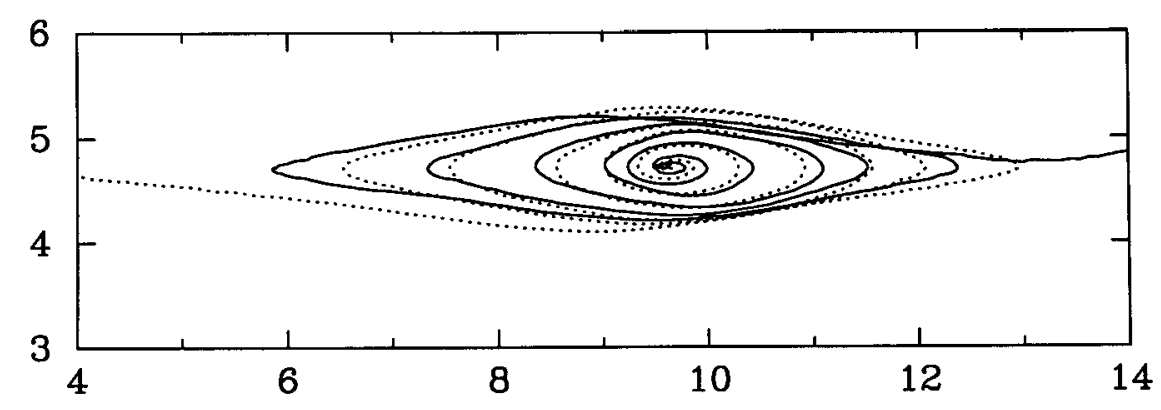

FIG. 11. Effect of mean zonal shear on vertical fragmentation. Trajectories of the upper- and lower-layer components of the vortex for $s=0.5, \lambda_{1}^{2}=0.03$, and $U_{0}=0.5$. The position of the vortex is defined as the location, scaled by the vortex radius $L$, of the maxima in the perturbation streamfunction $\psi-\psi_{0}$, evaluated at pressures of $690 \mathrm{mb}$ (solid line) and $3962 \mathrm{mb}$ (dotted line).

reaches approximately the radius of the vortex, the upper and lower sections of the vortex separate and are advected apart by the mean flow. Increasing the value of $\lambda_{1}^{2}$, that is, decreasing the radius of deformation or increasing the size of the vortex, decreases the rate at which the amplitude and period of the oscillations grow. Increasing $U_{0}$ has a similar effect. Stable oscillations occur for some parameter settings.

\section{e. Discussion}

The nonlinear behavior of nearly baroclinic, Gaussian vortices on an $f$ plane in a barotropic mean zonal shear can be broken down into five classifications: adjustment to a stable vertical structure with shedding of smaller vortices, adjustment to a stable vertical structure without shedding of smaller vortices, vertical fragmentation of the vortex preceded by oscillations in the position of the vortex, shearing of the vortex by the zonal flow, and stability. Figure 12 shows the regions of $\lambda_{1}^{2}-s$ parameter space over which each of these occurs for $U_{0}=0.5$.

Adjustment to a stable vertical structure with shedding of smaller vortices occurs over roughly the same region of $\lambda_{1}^{2}-s$ parameter space as horizontal fragmentation does in the absence of the zonal flow. Adjustment to a stable vertical structure without shedding of smaller vortices occurs in roughly the same region of $\lambda_{1}^{2}-s$ parameter space as tripole formation. Both of these occur only for vortices with diameters roughly larger than the first internal radius of deformation and with potential vorticity of opposite sign in the upper and lower layers. In the cases examined the initial vortex is not completely destroyed, although its vertical structure is modified.

Oscillations in the latitude and longitude of the vortex, usually followed by a breakup into separate upperand lower-layer vortices, occur only for vortices with diameters smaller than the first internal radius of deformation and with the same sign of vorticity at all altitudes. The amplitude and period of the oscillations grow as the upper- and lower-layer vortices move far- ther apart. When the separation becomes sufficiently large, the oscillations cease and the zonal flow advects the vortices away from each other. We have also found one example $\left(s=0.5, \lambda_{1}^{2}=0.1, U_{0}=0.5\right)$ in which the amplitude of the oscillations stops growing before the upper and lower vortices separate. It is interesting to note that these positional oscillations can occur for vortices with $s=0$, which can be represented by an equivalent barotropic model. The oscillations, however, cannot occur in the equivalent barotropic model, because they require the velocity to vary with altitude. Vortices that oscillate in longitude have been observed on Jupiter (Peek 1958; Reese and Smith 1966), Saturn (Reese 1971), and Neptune (Hammel et al. 1989). Comparison of the observations with our model will be discussed in section 6 .

Shearing of the vortex by the mean zonal flow occurs for vortices that are stable in the absence of the shear and have the opposite sign of vorticity as the shear flow. If the initial vortex has a different sign of vorticity at different altitudes, the part of the vortex rotating counter to the flow is sheared out. We also note that the unstable solutions that shed smaller vortices produce small, intense vortices, which have a vorticity of opposite sign to the vorticity of the mean zonal flow but are not sheared out by the flow. This indicates that a sufficiently strong vortex may exist in a counterrotating shear. This is consistent with calculations for the behavior of a patch of constant vorticity in a background flow with constant shear and strain (Moore and Saffman 1971; Kida 1981; Meacham et al. 1990), which show that counterrotating vortices are sheared out if the absolute value of their vorticity is less than about six times the vorticity of the mean flow.

Vortices that are stable in the absence of the mean zonal flow are also stable in a mean zonal shear with the same sign of vorticity as the vortex. While these vortices are stable, they are not necessarily steady: the aspect ratio and orientation of the vortex vary quasiperiodically. These oscillations are qualitatively similar to the nutating solutions found by Kida (1981) and 


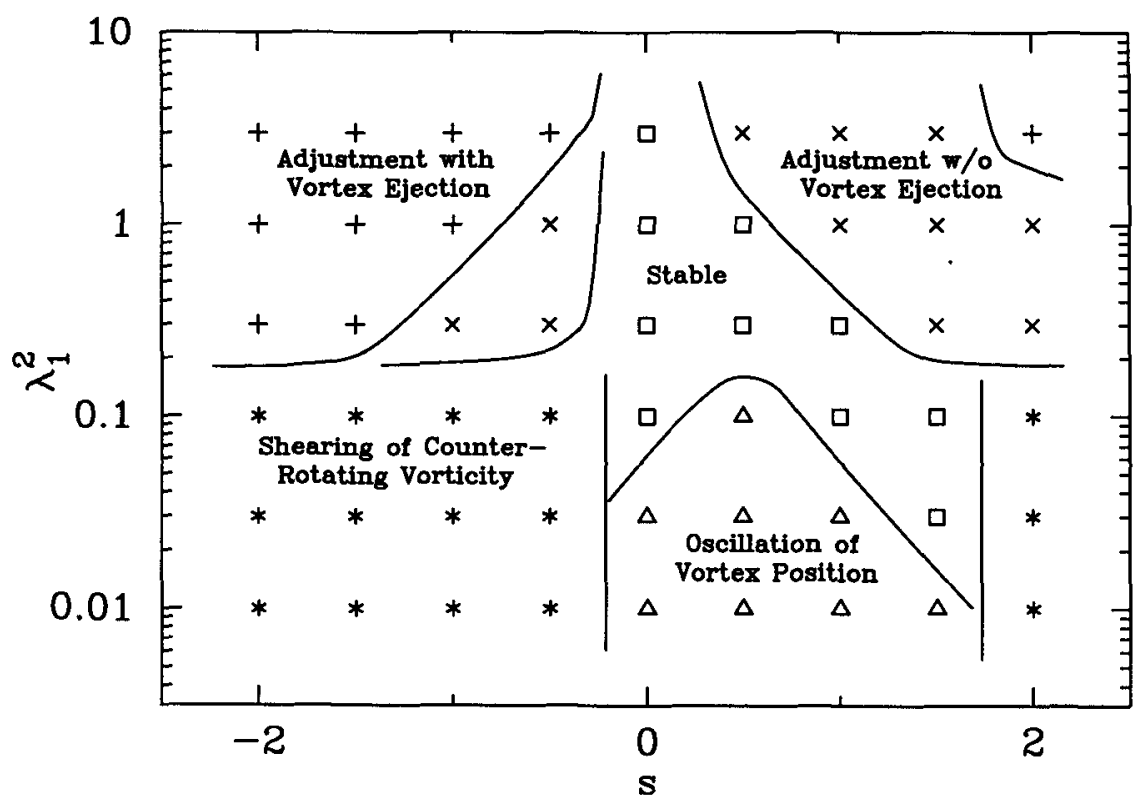

FIG. 12. Regime diagram for the behavior of vortices on an $f$-plane with mean zonal flow $U_{0}$ $=0.5$. The horizontal axis is $s$, the ratio of the second baroclinic mode to the first baroclinic mode in the initial vortex. The vertical axis is $\lambda_{1}^{2}$, the square of the radius of the vortex relative to the first internal radius of deformation. The open squares are stable vortices, the plus signs are vortices that adjust to stability while ejecting smaller vortices, the crosses are vortices that adjust to a stable state with a different vertical structure from the initial condition without ejecting smaller vortices, the asterisks are vortices that are sheared out at the altitudes at which they rotate counter to the mean flow, and the open triangles are vortices that develop oscillations in longitude and latitude.

Meacham et al. (1990) in a single-layer model and were used by Polvani et al. (1990) to explain the variations in shape of Neptune's Great Dark Spot. Our results indicate that periodic variations in the shape and orientation of vortices can occur in models with continuous vorticity distributions and nontrivial vertical structure. Oscillations of vortex shape in a model with a continuous vorticity distribution have also been seen by Marcus (1990). However, Marcus found that the oscillations in shape damped out in a few vortex turnaround times, provided that the background flow contained numerous small-scale filaments of vorticity (which our simulations do not have), resulting in a steady vortex.

\section{Effects of $\beta$}

We now consider the effects of a meridional gradient of the Coriolis parameter upon the evolution of vortices. The behavior of isolated, monopolar vortices on the $\beta$ plane in the absence of a mean zonal flow has received considerable attention. Using a linearized, quasigeostrophic, single-layer model, Flierl (1977) found that Rossby wave dispersion causes vortices to propagate westward and decay in amplitude, with the drift rate approaching the maximum Rossby wave speed at large times. Numerical simulations by Mc-
Williams and Flierl (1979) using a nonlinear, twomode, quasigeostrophic model show that nonlinear effects reduce the decay rate and allow meridional propagation of the vortex (equatorward for anticyclonic vortices, poleward for cyclonic vortices ). As the nonlinearity increases, or as the size of the vortex relative to the deformation radius increases, the propagation rate of the vortex relative to the fastest linear wave velocity increases. The limiting propagation speed is the maximum Rossby wave group velocity ( $-\beta / \lambda_{1}^{2}$ in the zonal direction, $-\beta / 4 \lambda_{1}^{2}$ in the meridional direction). They also found that with a purely baroclinic initial vortex, a vortex pair (dipole) spins up in the barotropic mode, resulting in a structure similar to the dipole modon with baroclinic rider found by Flierl et al. (1980). This can slow down the westward propagation of the baroclinic vortex or result in eastward propagation. A two-layer primitive equation model gives similar results (Mied and Lindemann 1979). Results from single-layer shallowwater models show that anticyclonic vortices propagate westward faster than cyclonic vortices (Davey and Killworth 1984; Williams and Yamagata 1984; McWilliams et al. 1986; Williams and Wilson 1988). Differences between cyclones and anticyclones cannot occur in a quasigeostrophic model, as the quasigeostrophic equations are invariant under the transformation $(x, y, \psi) \leftrightarrow$ $(x,-y,-\psi)$. 

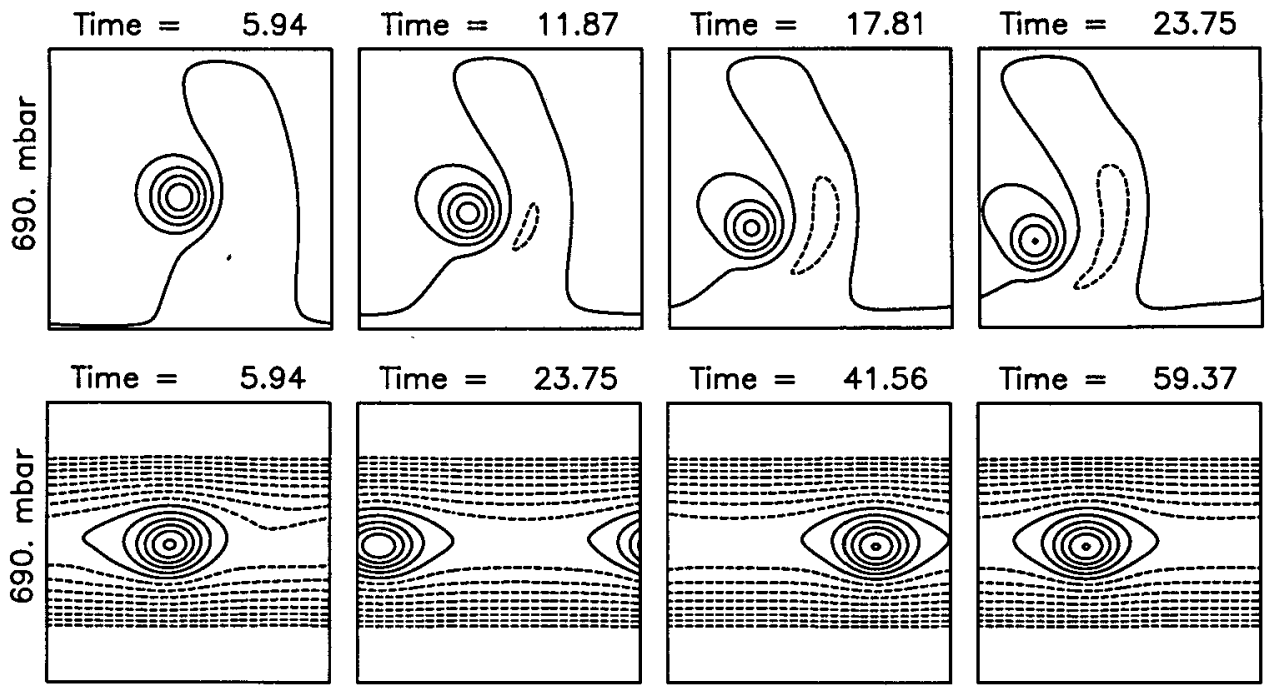

FIG. 13. Effect of mean zonal shear on a $\beta$-plane vortex. (a) Time evolution of the streamfunction at 690 $\mathrm{mb}$ of an $f$-plane vortex with $s=0.0, \lambda_{1}^{2}=1.0, U_{0}=0.0$, and $\beta=0.3$ at times 5.94, 11.87, 17.81, and 23.75. The contour interval is 0.25 , and negative contours are dashed. (b) Time evolution of the streanfunction at $690 \mathrm{mb}$ of an $f$-plane vortex with $s=0.0, \lambda_{1}^{2}=1.0, U_{0}=0.5$, and $\beta=0.3$ at times $5.94,23.75,41.56$, and 59.37. The contour interval is 0.25 , and negative contours are dashed. (b) Time evolution of the streamfunction at $690 \mathrm{mb}$ of an $f$ plane vortex with $s=0.0, \lambda_{1}^{2}=1.0, U_{0}=0.5$, and $\beta=0.3$ at times 5.94, 23.75, 41.56, and 59.37. The contour interval is 0.25 and negative contours are dashed. Contours for $\psi<-2.0$ are not shown.

The behavior of an isolated vortex on a quasigeostrophic $\beta$ plane is shown in Fig. 13a for a purely first baroclinic mode vortex with $s=0.0, \lambda_{1}^{2}=1.0, U_{0}$ $=0.0$, and $\beta=0.3$. The anticyclonic vortex drifts westward and equatorward and the peak amplitude of the streamfunction decays, with a weak radiation field

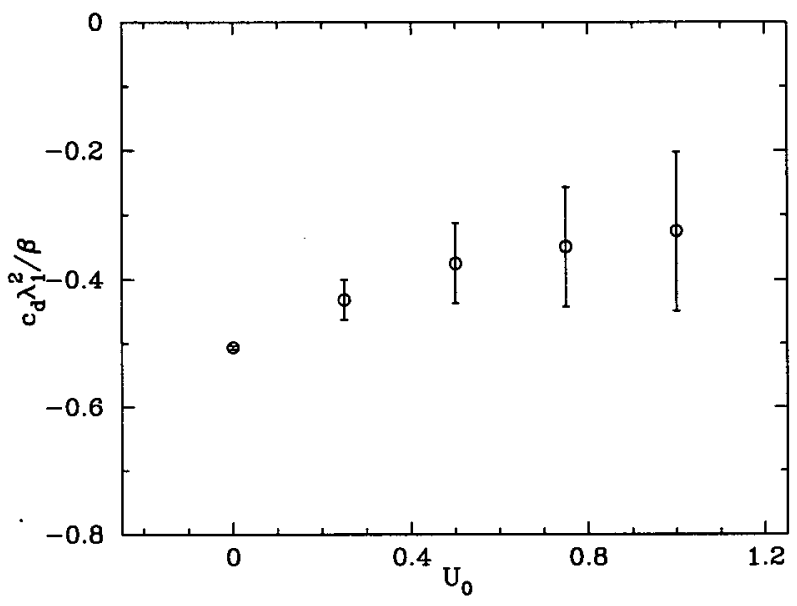

FIG. 14. Effect of changing $U_{0}$ on the propagation of vortices with $s=0.0, \lambda_{1}^{2}=1.0$, and $\beta=0.3$. Drift rate $c_{d}$ of the vortex relative to the mean zonal flow at the latitude of the maximum of the vortex streamfunction, normalized by the maximum Rossby wave speed $\beta \lambda_{1}^{-2}$, as a function of the strength of the mean zonal shear $U_{0}$. The errors are caused primarily by the uncertainty in the latitude of the vortex of plus or minus one-half of the grid spacing. forming east of the vortex. The zonal and meridional drift rates are -0.162 and -0.073 , respectively; the maximum amplitude Rossby wave group velocities are -0.3 zonally and -0.075 meridionally. The meridional drift rate of the vortex is very close to the maximum meridional Rossby wave speed, while the zonal drift rate of the vortex is only about half of the maximum zonal Rossby wave speed. In contrast to the two-mode model of McWilliams and Flierl (1979), our model does not allow a dipole to spin up in the barotropic mode because for a Jovian atmosphere with a deep fluid interior the barotropic mode is not affected by the baroclinic modes. We also do not see a spinup of a dipole in the second baroclinic mode; while a small disturbance forms in the second baroclinic mode, it is two orders of magnitude weaker than the first baroclinic mode.

If a mean zonal flow is present, the westward propagation still occurs, but the southward propagation and decay in amplitude are inhibited if the shear is sufficiently strong relative to the vorticity of the vortex or if the beta effect is sufficiently weak (e.g., Fig. 13b, with $\left.\psi_{0}=-\frac{1}{2} U_{0} y^{2}\right)$. In the presence of a mean zonal flow, the meridional propagation rate rapidly decreases to zero and the amplitude of the vortex oscillates instead of slowly decaying (the small amplitude oscillations also occur when $\beta=0.0$ and result from interactions with the mean zonal flow; they are accompanied by oscillations in the aspect ratio and orientation of the vortex as discussed in section $4 a$ ). Increasing the 
strength of the zonal flow decreases the meridional drift of the vortex and may also slightly decrease the zonal propagation rate relative to the mean flow (Fig. 14), although this is unclear due to the uncertainties in the drift rate. (The uncertainties are primarily due to the uncertainty in the latitude of the vortex, which creates an uncertainty in the mean zonal flow velocity at the center of the vortex.)

The drift rate of the vortex relative to the mean zonal flow is a roughly constant fraction of the maximum Rossby wave speed as $\beta$ varies. This is shown in Fig. 15 for $s=0.0, \lambda_{1}^{2}=1.0$, and $U_{0}=0.5$. Because the Rossby wave speed is proportional to $\beta$, the drift rate of the vortex in dimensional units is also roughly proportional to $\beta$. As $\beta$ is increased, the distance that the vortex drifts meridionally before assuming predominantly zonal motion increases, and the total amplitude decay of the vortex also increases. When $\beta$ becomes large enough, the interaction between the vortex and the mean zonal flow is no longer strong enough to balance the linear dispersion, and the zonal flow is no longer able to prevent meridional propagation and decay of the vortex.

Figure 16 shows the dependence of vortex drift rate on the size of the vortex relative to the first baroclinic deformation radius for $s=0.0, \beta=0.3$, and $U_{0}=0.5$. The drift rate of the vortex relative to the zonal flow becomes much smaller relative to the maximum Rossby wave speed as the size of the vortex relative to the radius of deformation decreases. This happens because the maximum Rossby wave speed is proportional to the square of the deformation radius; the drift rate nondimensionalized by the velocity scale of the vortex increases as the size of the vortex relative to the deformation radius decreases (Fig. 16b) . Decreasing $\lambda_{1}^{2}$ also

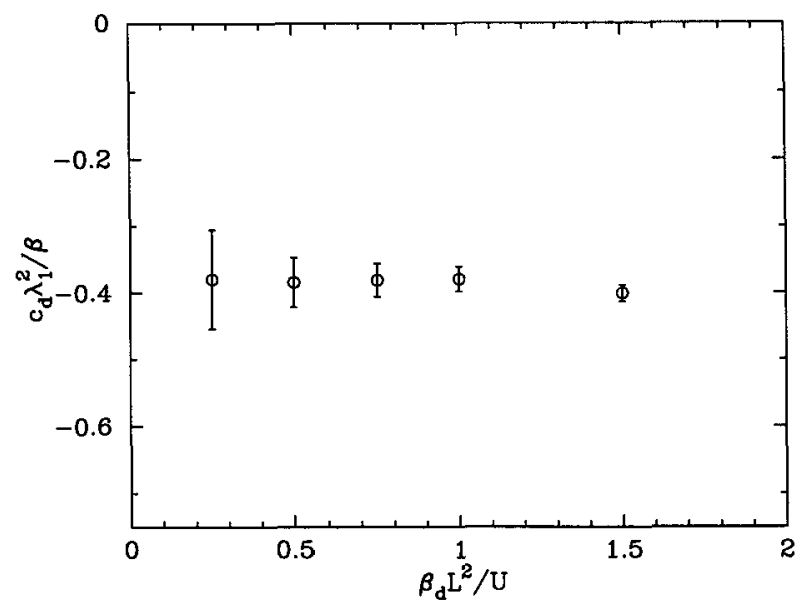

FIG. 15. Effect of changing $\beta$ on the propagation of vortices with $s=0.0, \lambda=1.0$, and $U_{0}=0.5$. Drift rate $c_{d}$ of the vortex relative to the mean zonal flow at the latitude of the peak of the vortex streamfunction, normalized by the maximum Rossby wave speed $\beta \lambda_{1}^{-2}$, as a function of $\beta$.
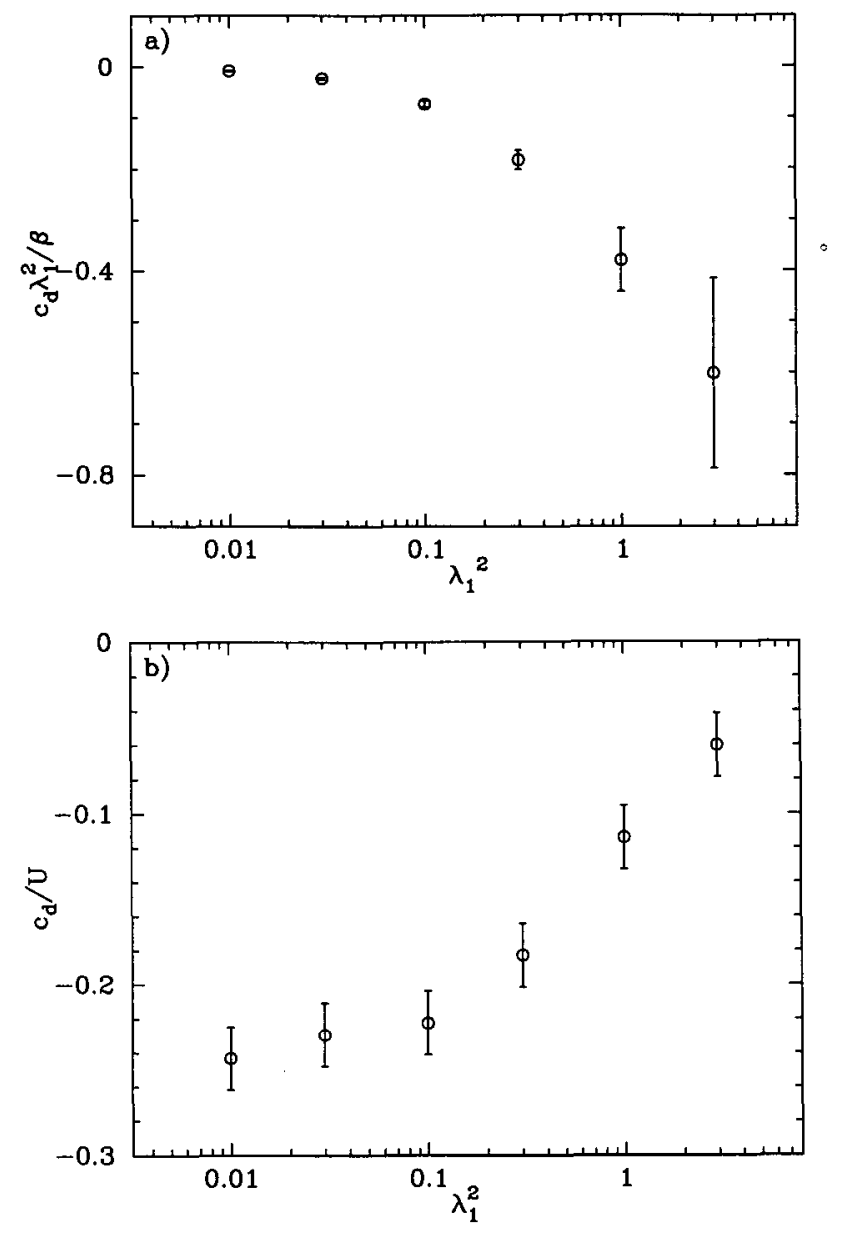

FIG. 16. Effect of changing $\lambda_{1}^{2}$ on the propagation of vortices with $s=0.0, U_{0}=0.5$, and $\beta=0.3$. (a) Drift rate of the vortex relative to the mean zonal flow at the latitude of the peak of the vortex streamfunction, normalized by the maximum Rossby wave speed $\beta \lambda_{1}^{-2}$, as a function of $\lambda_{1}^{2}$. (b) Drift rate of the vortex relative to the mean zonal flow at the latitude of the peak of the vortex streamfunction, normalized by the velocity scale of the vortex $U$, as a function of $\lambda_{1}^{2}$.

results in a greater meridional propagation of the vortex before its drift becomes zonal. The amplitude behavior as $\lambda_{1}^{2}$ changes is more complicated. For values of $\lambda_{1}^{2}$ $\geqslant 0.1$, the amplitude of the vortex oscillates with periods roughly of the order of vortex turnaround time due to interactions with the mean zonal flow but does not decay. At smaller values of $\lambda_{1}^{2}$, there is a gradual decay in amplitude, as well as long period variations in vortex amplitude corresponding with oscillations in the latitude of the vortex. These long period variations are caused by vertical fragmentation of the vortex, resulting in oscillations in the latitude and longitude of the vortex as discussed in section $4 \mathrm{~d}$.

We have also examined the effects of nonzero $\beta$ on the stability of vortices. For the baroclinically driven instabilities, the effect of a nonzero $\beta$ is westward prop- 

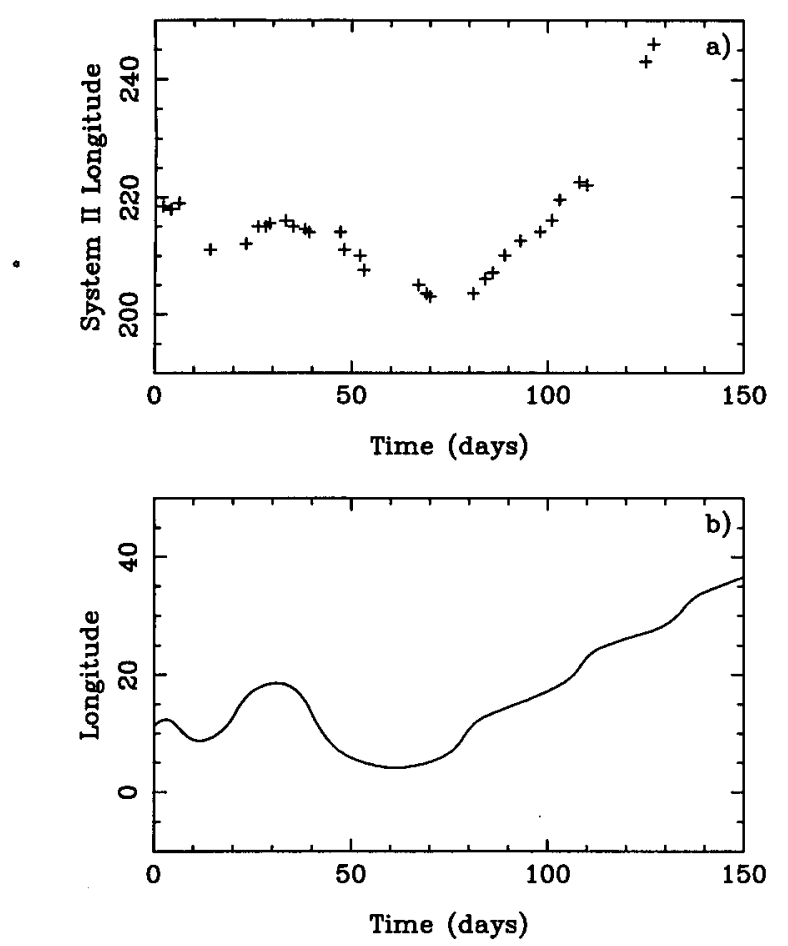

FIG. 17. (a) System II longitude variations of the oscillating spot of 1941-42 discussed by Peek (1958). (b) Variations in longitude of a model vortex with $s=0.5, \lambda_{1}^{2}=0.03, U_{0}=0.5$, and $\beta=0.1$. The results have been dimensionalized by a length scale of $2000 \mathrm{~km}$ and velocity scale of $50 \mathrm{~m} \mathrm{~s}^{-1}$, and the longitude has been converted to degrees of longitude at the latitude of the oscillating spot of 194142.

agation of the vortex as the instability occurs and the decay with time of any small or weak vortices produced by the instability. The form of the instability is otherwise unchanged. The effect of $\beta$ on vertical fragmentation is much more pronounced. Increasing $\beta$ results in more rapid growth of the instability: final breakup of a vortex with $s=0.5, \lambda_{1}^{2}=0.03$, and $U_{0}=0.5$ occurs at $t \approx 350$ for $\beta=0.0$, at $t \approx 200$ for $\beta=0.1$, and at $t \approx 100$ for $\beta=0.2$. With $\beta=0.3$ we also find very weak oscillations in latitude and longitude for some vortices with $\lambda_{1}^{2}=0.3$ that are stable when $\beta=0.0$, which would mean that the $\beta$ effect increases the maximum value of $\lambda_{1}^{2}$ for which vertical fragmentation occurs. However, the amplitude of the oscillations is only one or two times the grid spacing and may not be real. As discussed earlier in this section, vortices that are stable on the $f$ plane can also be stable and long-lived on the $\beta$ plane, provided that the mean zonal shear flow is strong enough for the nonlinear interaction between the vortex and the shear flow to balance linear Rossby wave dispersion. With the above caveats, the regime diagram for vortices in a mean zonal flow with the $\beta$ effect is the same as the regime diagram for $\beta=0.0$ (Fig. 12).

\section{Application to Jovian planets}

We will now compare the results of our numerical models to observations of the Jovian planets and use these comparisons to infer information about the vertical structure of Jovian atmospheres. In particular, we will use observations of longitudinal oscillations in the positions of vortices to estimate a lower limit on the deformation radius of Jupiter. We will also discuss the possibility of using measurements of vortex drift rates to place constraints on Jupiter's mean zonal flow below the level of the observed clouds.

\section{a. Longitudinal oscillation of vortex positions}

Vertical fragmentation by internal barotropic instability in the presence of a mean zonal shear results in oscillations of the latitude and longitude of the vortex. Several examples of vortices that oscillate in longitude are known. The most famous is Jupiter's Great Red Spot, which oscillates in longitude with a period of $89.89 \pm 0.11$ days and a mean amplitude of $0.77^{\circ}$ (Reese 1972). The oscillations persist even when the long-term drift of the GRS changes. Other examples include observations by Reese and Smith (1966) of a spot in Jupiter's North Temperate Belt that oscillated in longitude with a period of 300 days and a peak-topeak amplitude of $4^{\circ}$; an observation by Reese (1971) of a spot on Saturn that oscillated with a period of 169 days and a peak-to-peak amplitude of $16^{\circ}$ that decayed over time; two spots observed by Peek (1958) in 194041 and 1941-42, the first of which had a period of 72 days and a peak-to-peak amplitude of $20^{\circ}$ that decreased with time and the second of which had a similar period and amplitude except that the amplitude increased with time, and the second dark spot (D2) seen by Voyager 2 on Neptune that oscillated with a period of 36 days and a peak-to-peak amplitude of $97.8 \pm 0.9^{\circ}$ in longitude and $3.93 \pm 0.22^{\circ}$ in latitude (Hammel et al. 1989; Smith et al. 1989; Sromovsky 1991). Neptune's Great Dark Spot has also been observed to oscillate in latitude and longitude with a period of 106 days and peak-to-peak amplitudes of $10.7^{\circ}$ in longitude and $4.2^{\circ}$ in latitude (Sromovsky 1991 ).

The oscillating spot of 1941-42 (Peek 1958) shows the increase of period and amplitude with time that is seen with vertical fragmentation in a shear flow. Peek gives the size of the spot as "about the size of a satellite disk," which is on the order of $3000-5000 \mathrm{~km}$ depending upon the satellite, so we may estimate the length scale of the spot as $\sim 2000 \mathrm{~km}$. Using a typical velocity in Jupiter's atmosphere of $50 \mathrm{~m} \mathrm{~s}^{-1}$, this gives a time scale of $L / U=4 \times 10^{4} \mathrm{~s}$. The only full oscillation observed has a period of roughly 50-60 days and a peak-to-peak amplitude of about $15^{\circ}$, which gives a nondimensional period of approximately 100 and a nondimensional peak-to-peak amplitude of about 7 ; this is within the range of periods and amplitudes seen 
in our models. The amplitude and period of this oscillation are also increasing with time (Fig. 17). A detailed fit of the data to our models is not practical as the number of model parameters that affect the amplitude and period of the oscillation (vortex size, strength of the shear flow relative to the vortex, meridional gradient of mean potential vorticity, and vertical structure of the vortex) is larger than the number of observables (Peek gives only a time series of the longitude of the vortex and very rough indications of its size and latitude). The amplitude and period of oscillation also change with time for any given unstable vortex. If the oscillation in longitude of this spot is due to vertical fragmentation, then the diameter of the spot is a rough lower limit on the first internal radius of deformation, giving a lower limit to the deformation radius on Jupiter of $3000-5000 \mathrm{~km}$. This is at the upper end of the range of expected values for the deformation radius calculated by Ingersoll and Cuong (1981). Assuming that the deformation radius scales roughly as the square root of the water abundance as found by Achterberg and Ingersoll (1989), this would imply a water abundance in the interior relative to hydrogen on the order of 20 times the solar oxygen to hydrogen ratio. We also note that this gives static stabilities a few times larger than those assumed in calculating the interaction coefficients $\gamma_{i j k}$ used in our numerical model, although the interaction coefficients change only by $\sim 20 \%$ as the water abundance changes by two orders of magnitude (Achterberg and Ingersoll 1989).

The other observed oscillations in longitude described above cannot be completely explained by vertical fragmentation caused by internal barotropic instability. In the case of Neptune's second dark spot, the amplitude of the observed oscillations in latitude is roughly twice the latitudinal radius of the vortex; in our models, the upper- and lower-layer components separate completely when the latitudinal extent of the oscillations reaches the latitudinal radius of the vortex, and the oscillations stop. In addition, the amplitude of the observed oscillation in longitude is $\sim 9$ times the longitudinal radius of the vortex; in our models the largest observed oscillation in latitude is $\sim 5$ times the vortex radius.

For the remaining observed oscillations either the observed periods are too long or the amplitude of the oscillations decreases with time instead of growing. It is still interesting to speculate that these oscillations may be caused by variations in the position of the vortex with altitude caused by some unknown mechanism other than vertical fragmentation by instability. We have made preliminary attempts to model the behavior of stable vortices that have been strongly perturbed so that the initial position of the vortex varies with altitude. The results show oscillations in the latitude and longitude of the vortex. The amplitude of the oscillations decays to below the resolution of the model after a few times the oscillation period. This could explain observations of vortices that show decaying longitudinal oscillations, although matching the observed oscillation periods could be a problem. Polvani (1991) considered the problem of vortex alignment in a twolayer, $f$-plane, contour dynamics model with no mean zonal flow, in which he examined the evolution of an . initial condition with two patches of constant vorticity, one in each layer. He found that if the distance between the centers of the upper and lower vortex is large enough or small enough, or if the radius of the vortex is smaller than the deformation radius, the vortices will rotate around each other without their centers moving closer together, resulting in oscillations in the position of the vortex. At intermediate separations, the distance between the centers of the upper and lower vortices decreases while the vortices rotate around each other, resulting in oscillations in position in which the amplitude decreases with time. This suggests that if a vortex is perturbed such that its position varies with altitude, the latitude and longitude of the vortex at a given altitude will oscillate with time. The oscillations may or may not decay with time depending upon the size of the vortex and the amplitude of the perturbation. Whether or not similar behavior occurs with continuous vorticity distributions and a mean zonal flow, and whether or not plausible mechanisms exist for causing the initial variation in altitude of the position of the vortex are interesting topics for future research.

\section{b. Propagation of vortices}

In section 5 we found that the $\beta$ effect results in the westward propagation of vortices relative to the mean zonal flow. Drift rates of vortices have been measured from ground-based observations since the late $1800 \mathrm{~s}$ (Peek 1958) but the drift rates from these measurements cannot be compared to the velocity of the mean zonal flow since the propagation rates of the vortices themselves were used in determining the mean zonal flow velocity. Our models show that vortices can drift relative to the mean zonal flow by velocities on the order of the maximum Rossby wave speed. This will introduce errors when using the vortices to determine the velocity of the mean zonal flow. The error introduced by assuming that the vortices are simply advected by the zonal flow can be estimated by comparing the maximum zonal Rossby wave speed to the observed mean zonal flow velocities. The maximum Rossby wave speed is given by $\beta / \lambda_{1}^{2}$, which is simply $\beta$ times the square of the internal radius of deformation. On Jupiter, $\beta \approx 4.5 \times 10^{-12} \mathrm{~m}^{-1} \mathrm{~s}^{-1}$ at midlatitudes, and the radius of deformation has been estimated in the range from $500 \mathrm{~km}$ to $5000 \mathrm{~km}$ (Ingersoll and Cuong 1981). This gives a maximum Rossby wave speed, and therefore an estimate of maximum vortex drift rate relative to the zonal flow, in the range of $1-100 \mathrm{~m} \mathrm{~s}^{-1}$. The peak flow velocities in Jupiter's zonal jets vary from a few tens of meters per second to over $100 \mathrm{~m} \mathrm{~s}^{-1}$. 
Thus, if Jupiter's radius of deformation is near the lower end of the estimated range, the maximum speed at which vortices drift relative to the zonal flow is only a few percent of the mean zonal velocity. If, however, the radius of deformation is fairly large, it is possible for vortices to drift relative to the mean zonal flow at a large fraction of the mean zonal flow velocity.

Using observations from the Voyager imaging experiment, it is possible to measure the mean zonal flow velocities with good latitudinal resolution by tracking individual small cloud features (Ingersoll et al. 1981; Limaye 1986) instead of large spots. Dowling and Ingersoll (1988) measured the drift rates of the GRS and White Oval BC over the time interval between the Voyager 1 and Voyager 2 encounters. They obtained drift rates relative to System III (the reference frame determined by the rotation rate of Jupiter's magnetic field) of $-3.49 \mathrm{~m} \mathrm{~s}^{-1}$ and $4.84 \mathrm{~m} \mathrm{~s}^{-1}$, respectively. The mean zonal flow velocities in System III at the latitudes of the peaks in the streamfunction of the GRS (about $22.5^{\circ} \mathrm{S}$ planetographic latitude) and $\mathrm{BC}$ (about $33.5^{\circ} \mathrm{S}$ ) as measured by Limaye (1986) are -25 and -10 $\mathrm{m} \mathrm{s}^{-1}$, respectively, which implies that both the GRS and $\mathrm{BC}$ are propagating eastward with respect to the mean zonal flow. This contrasts with the results of section 5 that the $\beta$ effect results in westward propagation of vortices.

Using the mean zonal wind profile from Limaye (1986), the latitudes at which the mean zonal velocity is equal to the drift rate of the GRS and BC are $23.5^{\circ} \mathrm{S}$ and $34.5^{\circ} \mathrm{S}$, respectively. The former is still within the latitude range of the quiescent central part of the GRS, which makes locating the latitude of the streamfunction peak somewhat difficult, so that although the GRS may be moving eastward relative to the mean zonal flow the data are inconclusive. However, the latitude at which the mean zonal flow has the same velocity as the drift rate of $\mathrm{BC}$ is clearly south of the peak in the streamfunction of $\mathrm{BC}$. Thus, it appears that $\mathrm{BC}$ propagates to the east with respect to the mean zonal flow.

There are at least two possible explanations for the eastward drift of White Oval BC. First, there is a class of known steady solutions to the quasigeostrophic equations on the $\beta$ plane that have eastward propagation speeds-the "dipolar modon with baroclinic rider" discussed by Flierl et al. (1980). However, these solutions were obtained in the absence of a mean zonal flow and involve a dipolar structure in one of the vertical modes, which is unlikely to survive in a shear flow. Also, the numerical solutions by McWilliams and Flierl (1979) suggest that the modons with rider solutions are not stable. Another possible explanation is that the meridional gradient of the background mean zonal potential vorticity is negative. In general, either vertical shear or meridional curvature of the mean zonal flow, both of which create a background potential vorticity gradient, can create effects such as Rossby waves that are similar to those caused by nonzero $\beta$ (Gill 1982).
The important parameter is the meridional gradient of the background potential vorticity, which reduces to $\beta$ in the absence of a mean zonal flow.

To determine if potential vorticity gradients from curvature of the zonal flow have a similar effect upon vortex propagation as latitudinal gradients in the Coriolis parameter, we performed simulations with a flow in the barotropic mode of the form

$$
\psi_{0}(y)=-\left(\frac{U_{0}}{2}\right) y^{2}+\left(\frac{B}{6}\right) y^{3}
$$

which has a meridional potential vorticity gradient of $B$, for $s=0.0, \lambda_{1}^{2}=1.0, \beta=0.0, U_{0}=0.5$, and $B$ $= \pm 0.3$. The vortex with $B=0.3$ propagates to the south and west, while the vortex with $B=-0.3$ propagates to the north and east. The drift rates of the vortex relative to the mean zonal flow, nondimensionalized by the velocity scale $U$, are $-0.13 \pm 0.03$ for $B=0.3$ and $+0.13 \pm 0.03$ for $B=-0.3$. These values can be compared to the value of $-0.11 \pm 0.02$ for the case with $\beta$ $=0.3$ and $B=0$. Thus, a meridional potential vorticity gradient caused by curvature of the mean zonal flow has approximately the same effect on the propagation of a vortex as does a potential vorticity gradient caused by the latitudinal variation of the Coriolis parameter. Furthermore, a negative meridional potential vorticity gradient gives vortices that propagate eastward relative to the mean zonal flow.

There is some observational evidence that the potential vorticity gradient is negative at the latitudes of the GRS and White Oval BC. Figure 5 of Dowling and Ingersoll (1989) shows a negative potential vorticity gradient from $-35^{\circ} \mathrm{S}$ to $-31.5^{\circ} \mathrm{S}$ and from $-25^{\circ} \mathrm{S}$ to $-17.5^{\circ} \mathrm{S}$; these latitude ranges include the centers of the GRS and White Oval BC. Because the zonal velocity below the observed cloud layer cannot be measured directly, Dowling and Ingersoll used observations of vortex tube stretching to infer the latitudinal mean zonal pressure gradient, and thus the mean zonal velocity, of the deep lower layer. Nevertheless, the sign of the potential vorticity gradient observed by Dowling and Ingersoll is consistent with the model results and the observation that the GRS and White Oval BC are propagating eastward with respect to the mean zonal flow.

\section{Conclusions}

Numerical simulations of the nonlinear stability of baroclinic Jovian vortices on the $f$ plane indicate that stable vortices, or vortices that appear to be stable when seen only at the level of the observed cloud deck, exist over the range of sizes examined, with radii varying from one-tenth deformation radius to about twice the deformation radius. Although we did not model larger vortices for computational reasons (the number of grid points required to adequately resolve the deformation 
radius of the second baroclinic mode becomes prohibitively large for $\lambda_{1}^{2}>3.0$ ), previous studies show that barotropic vortices larger than twice the deformation radius are linearly stable in a two-layer model (Flierl 1988 ) and are nonlinearly stable in a barotropic shallow-water model (e.g., Williams and Wilson 1988; Dowling and Ingersoll 1989). This matches observations of Jupiter, which show vortices with diameters ranging from a few hundred kilometers to over twenty thousand that persist for months to centuries. The tendency of a nonzero $\beta$ to cause meridional propagation and decay of vortices can be countered by a sufficiently strong mean zonal flow, allowing vortices to persist for fairly long times, even in the presence of meridional potential vorticity gradients.

Horizontal fragmentation of vortices by instabilities is seen only for vortices with diameters larger than the first internal radius of deformation. We should note, however, that vortices of any size with radial streamfunction profiles steeper than the Gaussian profiles used in our model can experience horizontal fragmentation through standard barotropic instability; the result, in the absence of a mean zonal flow, is fragmentation into dipoles, with each component of the dipole having the same vertical structure (Gent and McWilliams 1986).

Internal barotropic instability, which occurs for vortices smaller than the deformation radius, can be detected only from observations of cloud top motions by its effect on the position of the vortex. In the presence of a mean zonal flow, it results in the oscillation of the position of the vortex in both latitude and longitude. This instability may explain some, but not all, observations of vortices that oscillate in longitude, in particular the oscillating vortex of 1941-42 on Jupiter. If the latter is truly an example of internal barotropic instability, it implies that the deformation radius on Jupiter is near the upper limit of the range estimated by Ingersoll and Cuong (1981), in the range of around 3000$5000 \mathrm{~km}$, which is surprisingly large.

The presence of a meridional potential vorticity gradient results in the propagation and decay of vortices. The decay can be inhibited by a sufficiently strong zonal flow, although the vortices will drift zonally relative to the mean flow. If the deformation radius is large, the drift rate relative to the mean flow may be a large fraction of the mean flow velocity, which will introduce errors when using observed vortex drift rates as estimates of the mean zonal flow velocities. The direction that vortices drift relative to the mean flow depends upon the sign of the meridional potential vorticity gradient. Thus, measurements of vortex drift rates may be helpful in constraining models for the flow beneath the observed cloud layer; measurement of drift rates for a number of vortices at different latitudes would be useful.

Acknowledgments. Most of the computations in this paper were performed on the Cray Y/MP of the San
Diego Supercomputer Center. This research was supported by the NASA Planetary Atmospheres program under Grant NAGW-1956 and the Neptune Data Analysis Program under Grant NAGW-2363.

\section{REFERENCES}

Achterberg, R. K., and A. P. Ingersoll, 1989: A normal-mode approach to Jovian atmospheric dynamics. J. Atmos. Sci., 46, $2448-2462$.

Arakawa, A., 1966: Computational design for long-term numerical integrations of the equations of atmospheric motion. J. Comput. Phys., 1, 119-143.

Conrath, B. J., P. J. Gierasch, and N. Nath, 1981: Stability of zonal flows on Jupiter. Icarus, 48, 256-282.

Davey, M. K., and P. D. Killworth, 1984: Isolated waves and eddies in a shallow water model. J. Phys. Oceanogr., 14, 1047-1064.

Dowling, T. E., and A. P. Ingersoll, 1988: Potential vorticity and layer thickness variations in the flow around Jupiter's Great Red Spot and White Oval BC. J. Atmos. Sci., 45, 1380-1396.

$\ldots$, and — 1989: Jupiter's Great Red Spot as a shallow water system. J. Atmos. Sci., 46, 3256-3278.

Flierl, G. R., 1977: The application of linear quasigeostrophic dynamics to Gulf Stream Rings. J. Phys. Oceanogr., 7, 365-379. , 1988: On the instability of geostrophic vortices. J. Fluid Mech., 197, 349-388.

, V. D. Larichev, J. C. McWilliams, and G. M. Reznik, 1980: The dynamics of baroclinic and barotropic solitary eddies. Dyn. Atmos. Oceans, 5, 1-41.

Gent, P. R., and J. C. McWilliams, 1986: The instability of barotropic circular vortices. Geophys. Astrophys. Fluid Dyn., 35, 209-233.

Gierasch, P. J., A. P. Ingersoll, and D. Pollard, 1979: Baroclinic instabilities in Jupiter's zonal flow. Icarus, 40, 205-212.

Gill, A. E., 1982: Atmosphere-Ocean Dynamics. Academic Press, 662 pp.

Haltiner, G. J., and R. T. Williams, 1980: Numerical Prediction and Dynamic Meteorology. $2 \mathrm{~d}$ ed. John Wiley and Sons, $477 \mathrm{pp}$.

Hammel, H. B., R. F. Beebe, E. M. DeJong, C. J. Hansen, C. D. Howell, A. P. Ingersoll, T. V. Johnson, S. S. Limaye, J. A. Magalhães, J. B. Pollack, L. A. Sromovsky, V. E. Suomi, and C. E. Swift, 1989: Neptune's wind speeds obtained by tracking clouds in Voyager images. Science, 245, 1367-1369.

Hatzes, A., D. D. Wenkert, A. P. Ingersoll, and G. E. Danielson, 1981: Oscillations and velocity structure of a long-lived cyclonic spot. J. Geophys. Res., 86, 8745-8749.

Helfrich, K. R., and U. Send, 1988: Finite-amplitude evolution of two-layer geostrophic vortices. J. Fluid Mech., 197, 331-348.

Hide, R., 1961: Origin of Jupiter's Great Red Spot. Nature, 190, 895896.

Holton, J. R., 1979: An Introduction to Dynamic Meteorology. 2d ed. Academic Press, 391 pp.

Ikeda, M., 1981: Instability and splitting of mesoscale rings using a two-layer quasigeostrophic model on an f-plane. $J$. Phys. Oceanogr., 11, 987-998.

Ingersoll, A. P., 1969: Inertial Taylor columns and Jupiter's Great Red Spot. J. Atmos. Sci., 26, 744-752.

, and P. G. Cuong, 1981: Numerical model of long-lived Jovian vortices. J. Atmos. Sci., 38, 2067-2076.

- R. F. Beebe, J. L. Mitchell, G. W. Garneau, G. M. Yagi, and J.-P. Müller, 1981: Interaction of eddies and mean zonal flow on Jupiter as inferred from Voyager 1 and 2 images. J. Geophys. Res., 86, 8733-8743.

- R. F. Beebe, B. J. Conrath, and G. E. Hunt, 1984: Structure and dynamics of Saturn's atmosphere. Saturn, T. Gehrels and M. S. Matthews, Eds., University of Arizona Press, 195-238.

Kida, S., 1981: Motion of an elliptic vortex in a uniform shear flow. J. Phys. Soc. Japan, 50, 3517-3520.

Limaye, S. S., 1986: Jupiter: New estimates of the mean zonal flow at the cloud level. Icarus, 65, 335-352. 
Mac Low, M.-M., and A. P. Ingersoll, 1986: Merging of vortices in the atmosphere of Jupiter: An analysis of Voyager images. Icarus, 65, 353-369.

Marcus, P. S., 1988: A numerical simulation of the Great Red Spot of Jupiter. Nature, 331, 693-696.

- 1990: Vortex dynamics in a shearing zonal flow. J. Fluid Mech., 215, 393-430.

Maxworthy, T., and L. G. Redekopp, 1976: A solitary wave theory of the Great Red Spot and other observed features in the Jovian atmosphere. Icarus, 29, 261-271.

McWilliams, J. C., and G. R. Flierl, 1979: On the evolution of isolated, nonlinear vortices. J. Phys. Oceanogr., 9, 1155-1182.

- P. R. Gent, and N. J. Norton, 1986: The evolution of balanced, low-mode vortices on the $\beta$-plane. J. Phys. Oceanogr., 16, 838855.

Meacham, S. P., G. R. Flierl, and U. Send, 1990: Vortices in shear. Dyn. Atmos. Oceans, 14, 333-386.

Meyers, S. D., J. Sommeria, and H. L. Swinney, 1989: Laboratory study of the dynamics of Jovian-type vortices. Physica $D, 37$, 515-530.

Mied, R. P., and G. J. Lindemann, 1979: The propagation and evolution of cyclonic Gulf Stream rings. J. Phys. Oceanogr., 9, 1183-1206.

Mitchell, J. L., R. F. Beebe, A. P. Ingersoll, and G. W. Garneau, 1981: Flow fields within Jupiter's Great Red Spot and White Oval BC. J. Geophys. Res., 86, 8751-8757.

Moore, D. W., and P. G. Saffman, 1971: Structure of a line vortex in an imposed strain. Aircraft Wake Turbulence and its Detection, J. H. Olsen, A. Goldburg, and M. Rogers, Eds., Plenum, 339-354.

Nezlin, M. V., A. Yu. Rylov, A. S. Trubnikov, and A. V. Khutorestskii, 1990: Cyclonic-anticyclonic asymmetry and a new soliton concept for Rossby vortices in the laboratory, oceans and the atmospheres of the giant planets. Geophys. Astrophys. Fluid Dyn., 52, 211-247.

Pedlosky, J., 1987: Geophysical Fluid Dynamics. 2d ed. SpringerVerlag, $710 \mathrm{pp}$.
Peek, B. M., 1958: The Planet Jupiter. Faber and Faber, 283 pp.

Polvani, L. M., 1991: Two-layer geostrophic vortex dynamics. Part 2. Alignment and two-layer V-states. J. Fluid Mech., 221, 241270.

_ J. Wisdom, E. DeJong, and A. P. Ingersoll, 1990: Simple dynamical models of Neptune's Great Dark Spot. Science, 249, 1393-1398.

Read, P. L., 1986: Stable, baroclinic eddies on Jupiter and Saturn: A laboratory analog and some observational tests. Icarus, 65, 304334.

__ , and R. Hide, 1983: Long-lived eddies in the laboratory and in the atmospheres of Jupiter and Saturn. Nature, 302, 126-129.

- $\ldots$ and - 1984: An isolated baroclinic eddy as a laboratory analogue of the Great Red Spot on Jupiter. Nature, 308, 45-48.

Reese, E. J., 1971: Recent photographic measurements of Saturn. Icarus, 15, 466-479.

_, 1972: Jupiter: its red spot and disturbances in 1970-1971. Icarus, 17, 57-72.

—_, and B. A. Smith, 1966: A rapidly moving spot on Jupiter's north temperate belt. Icarus, 5, 248-257.

Smith, B. A., and collaborators, 1979a: The Jupiter system through the eyes of Voyager 1. Science, 204, 951-971.

—_, and collaborators, 1979b: The Galilean satellites and Jupiter: Voyager 2 imaging science results. Science, 206, 927-950.

- - , and collaborators, 1989: Voyager 2 at Neptune: Imaging science results. Science, 246, 1422-1449.

Sommeria, J., S. D. Meyers, and H. L. Swinney, 1988: A laboratory simulation of the Great Red Spot of Jupiter. Nature, 331, 689693.

Sromovsky, L. A., 1991: Latitudinal and longitudinal oscillations of cloud features on Neptune. Science, 254, 684-686.

Stevenson, D. J., 1982: Interiors of the giant planets. Ann. Rev. Earth Planet. Sci., 10, 257-295.

Williams, G. P., and T. Yamagata, 1984: Geostrophic regimes, intermediate solitary vortices and Jovian eddies. J. Atmos. Sci., 41, 453-478.

_- and R. J. Wilson, 1988: The stability and genesis of Rossby vortices. J. Atmos. Sci., 45, 207-241. 